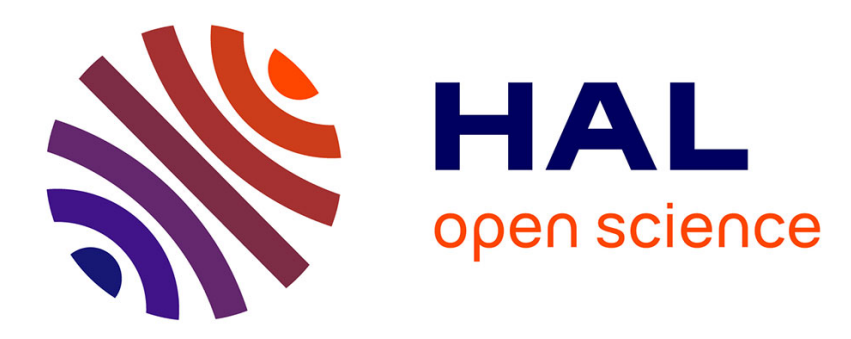

\title{
The matrix approach for abstract argumentation frameworks
}

Claudette Cayrol, Yuming Xu

\section{To cite this version:}

Claudette Cayrol, Yuming Xu. The matrix approach for abstract argumentation frameworks. [Research Report] RR- -2015-01- -FR, IRIT: Institut en Recherche Informatique de Toulouse. 2015. hal-03190216

\section{HAL Id: hal-03190216 https://hal.science/hal-03190216}

Submitted on 7 Apr 2021

HAL is a multi-disciplinary open access archive for the deposit and dissemination of scientific research documents, whether they are published or not. The documents may come from teaching and research institutions in France or abroad, or from public or private research centers.
L'archive ouverte pluridisciplinaire HAL, est destinée au dépôt et à la diffusion de documents scientifiques de niveau recherche, publiés ou non, émanant des établissements d'enseignement et de recherche français ou étrangers, des laboratoires publics ou privés. 


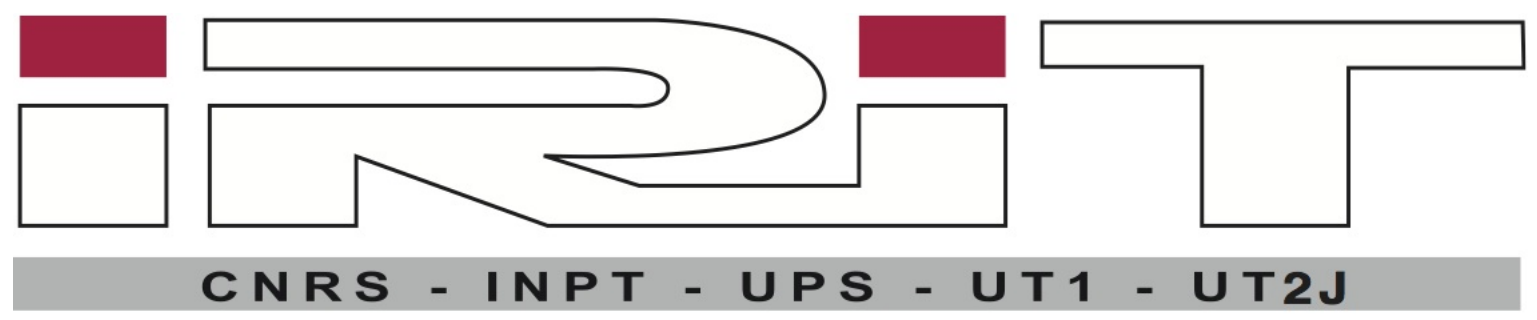

The matrix approach for abstract argumentation frameworks

Claudette CAYROL,

Yuming XU

IRIT Report

RR- -2015-01- -FR

February 2015 



\begin{abstract}
The matrices and the operation of dual interchange are introduced into the study of Dung's theory of argumentation frameworks. It is showed that every argumentation framework can be represented by a matrix, and the basic extensions (such as admissible, stable, complete) of an argumentation framework can be determined by sub-blocks of its matrix. In particular, an efficient approach for determining the basic extensions has been developed using two types of partition of a matrix. Furthermore, we develop the topic of matrix reduction along two different lines. The first one enables to contract parts of the matrix into a single argument, thus reducing the matrix into a less order matrix playing the same role for the determination of extensions. The second kind of reduction enables to decompose an extension into several extensions of different sub-argumentation frameworks. It makes us not only solve the problem of determining grounded and preferred extensions, but also obtain results about dynamics of argumentation frameworks which are better than the main results by Liao and Koons[19].
\end{abstract}




\section{Contents}

1 Introduction 1

2 Background on abstract argumentation frameworks 2

3 The matrix representation 4

4 Characterizing the extensions of an $A F \quad 6$

4.1 Characterizing the conflict-free subsets . . . . . . . . . . . . 6

4.2 Characterizing the stable extensions . . . . . . . . . . . . . 7

4.3 Characterizing the admissible subsets . . . . . . . . . . 8

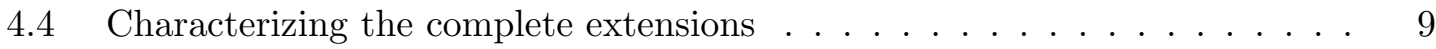

4.5 The standard forms of the matrix $M(A F) \ldots \ldots \ldots \ldots$

5 Matrix reduction based on the contraction of $A F \quad 14$

5.1 The first type of reduction . . . . . . . . . . . . . . . . . . . 14

5.2 The second type of reduction . . . . . . . . . . . . . 16

5.3 The third type of reduction . . . . . . . . . . . . 18

6 Matrix reduction based on the division of $A F \quad 20$

6.1 Building the grounded and preferred extensions . . . . . . . . . . . 20

6.2 Handling the dynamics argumentation framework $\ldots \ldots \ldots . \ldots . \ldots 23$

7 Concluding remarks and future works 28

$\begin{array}{ll}\text { A Appendix: Proofs } & 29\end{array}$

B References $\quad 33$ 


\section{Introduction}

In recent years, the area of argumentation begins to become increasingly central as a core study within Artificial Intelligence. A number of papers investigated and compared the properties of different semantics which have been proposed for abstract argumentation frameworks as introduced by Dung[14, 1, 2, 3, 7, 16, 25]. It is well known that abstract argumentation frameworks are usually represented as directed graphs, which play a significant role in modeling and analyzing the extension-based semantics of argumentation frameworks. For further notations and techniques of argumentation, we refer the reader to $[14,4,23,25]$.

Directed graphs have been widely used for modeling and analyzing argumentation frameworks because of the feature of visualization[13, 15, 1, 11, 12]. Furthermore, the labeling and game approach developed by Sanjay Modgil and Martin Caminada [7, 8, 20, 21] respectively are two excellent methods for the proof theories and algorithms of argumentation frameworks. In this paper, we propose another novel idea, that is, the matrix representation of argumentation frameworks. As we know, matrix theory is an important mathematic tool used in particular for representing and handling graphs, so the potential application to argumentation frameworks is worth to expect.

Our aim is to introduce matrices and the operation of dual interchange into the study of argumentation frameworks so as to propose new efficient approaches for determining traditional extensions. First, we assign a matrix of order $n$ for each argumentation framework with $n$ arguments. Each element of the matrix has only two possible values: 1 and 0 , where 1 represents the attack relation and 0 represents the non-attack relation between two arguments. Under this assignment, the matrix can be thought as a representation of the argumentation framework. This representation enables to establish links between extensions (under various semantics) of the argumentation framework and the internal structure of the matrix, namely sub-blocks of the matrix. Moreover, the matrix of an argumentation framework can be turned into a standard form, from which the determination of admissible and complete extensions can be easily achieved through checking some sub-blocks of this standard form. Furthermore, we propose the reduced matrix wrt conflict-free subsets, by which the determination of various extensions becomes more efficient. The idea is to contract a conflict-free subset into a single argument, thus reducing the argumentation framework into another one with less arguments, which plays the same role for the determination of extensions. This approach has not been mentioned in the literature as we know. Finally, we present the reduced matrix wrt extensions and give the decomposition theory for extensions. It can be used to handle the extensions with minimality and maximality, for example, to determine the preferred extensions. It can also be related to the topic of directionality and enables us to obtain results about dynamics of argumentation frameworks, which are better than the main results by Liao and Koons[19].

The report is organized as follows. Section 2 recalls the basic definitions on abstract argumentation. Section 3 introduces the matrix representation of argumentation frame- 
works and the operation of dual interchange of matrices. This operation makes the matrix representation be a powerful tool for the study of argumentation frameworks. Section 4 describes the characterization theorems for stable, admissible and complete extensions. Furthermore, we integrate these theorems and obtain two kinds of standard forms for matrices by dual interchange. Section 5 presents the reduced matrix of an argumentation framework with respect to conflict-free subsets. We discuss three basic types of reduced matrix depending on the number of conflict-free subsets and the relationship between them. Section 6 proposes the division-based reduction and its applications. We first consider the determination of grounded and preferred extensions. Then, we consider an application to the study of dynamics of argumentation frameworks. Two types of updated argumentation frameworks are considered corresponding to the addition and the removal of arguments (or attacks). All the proofs are given in Appendix except for the trivial ones.

\section{Background on abstract argumentation frameworks}

In this section, we recall the basic notions of abstract argumentation framework[14, 23].

Definition 1 An abstract argumentation framework is a pair $A F=(A, R)$, where $A$ is a finite set of arguments and $R \subseteq A \times A$ represents the attack relation. For any $S \subseteq A$, we say that $S$ is conflict-free if there are no $a, b \in S$ such that $(a, b) \in R ; a \in A$ is attacked by $S$ if there is some $b \in S$ such that $(b, a) \in R ; a \in A$ attacks $S$ if there is some $b \in S$ such that $(a, b) \in R ; a \in A$ is defended by (or acceptable wrt) $S$ if for each $b \in A$ with $(b, a) \in R$, we have that $b$ is attacked by $S$.

An argumentation framework has an obvious representation as a directed graph where nodes are arguments and edges are drawn from attacking to attacked arguments. For example, the argumentation framework $(A, R)$ with $A=\{a, b, c\}$ and $R=\{(a, b),(a, c)\}$ is represented by the following directed graph:

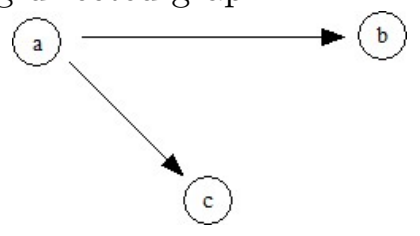

The following notations are inspired from graph theory and will be used in the following.

Notations Let $A F=(A, R)$ be an argumentation framework and $S \subseteq A$.

- $R^{+}(S)$ denotes the set of arguments attacked by $S$

- $R^{-}(S)$ denotes the set of arguments attacking $S$

- $I_{A F}$ denotes the set of arguments which are not attacked (also called initial arguments of $A F$ ) 
An argumentation semantics is the formal definition of a method ruling the argument evaluation process. Two main styles of argumentation semantics definition can be identified in the literature: extension-based and labelling-based. In the extension-based approach a semantics definition specifies how to derive from an argumentation framework a set of extensions, where an extension $E$ of an argumentation framework $(A, R)$ is simply a subset of A, intuitively representing a set of arguments which can survive together or are collectively acceptable. In the labelling-based approach a semantics definition specifies how to derive from an argumentation framework a set of labellings, where a labelling $\mathrm{L}$ is the assignment to each argument in A of a label taken from a predefined set L, which corresponds to the possible alternative states of an argument in the context of a single labelling. Here, we only recall the common extension-based semantics of $A F$.

Definition 2 Let $A F=(A, R)$ be an argumentation framework and $S \subseteq A$.

- $S$ is a stable extension of $A F$, if $S$ is conflict-free and each $a \in A \backslash S$ is attacked by $S$.

- $S$ is admissible in $A F$, if $S$ is conflict-free and each $a \in S$ is defended by $S$. For convenience, we denote the collection of all admissible subsets in $A F$ by $a(A F)$.

- $S$ is a preferred extension of $A F$, if $S \in a(A F)$ and $S$ is a maximal element (wrt set inclusion) of $a(A F)$.

- $S$ is a complete extension of $A F$, if $S \in a(A F)$ and for each a $\in A$ defended by $S$, we have $a \in S$. For convenience, we denote the collection of all complete extensions by $c(A F)$.

- $S$ is the grounded extension of $A F$, if $S \in c(A F)$ and $S$ is the least element (wrt set inclusion) of $c(A F)$.

The common extension-based semantics can be characterized in terms of subsets of attacked/attacking arguments, due to the following results:

Proposition 1 Let $A F=(A, R)$ be an argumentation framework and $S \subseteq A$.

- $S$ is conflict-free iff $S \cap R^{+}(S)=\emptyset$ iff $R^{+}(S) \subseteq A \backslash S$

- $S$ is stable iff $R^{+}(S)=A \backslash S$

- $S$ is admissible iff $R^{-}(S) \subseteq R^{+}(S) \subseteq A \backslash S$

Note that any stable extension must be complete and any complete extension must be admissible. Since every extension under the standard semantics (stable, complete, preferred and grounded) introduced by Dung is an admissible set, the concept of admissible set plays an important role in the study of argumentation frameworks. 
Remark 1 In fact, the grounded extension can also be defined by the characteristic function. Let $A F=(A, R)$, the function $\mathcal{F}_{A F}: 2^{A} \rightarrow 2^{A}$ which, given a set $S \subseteq A$, returns the set of the acceptable arguments wrt $S$, is called the characteristic function of $A F$. The grounded extension of $A F$ is the least fixed point of $\mathcal{F}_{A F}$.

In Section 6, we will discuss some properties of argumentation frameworks related to the directionality. So, we need to recall the definition of sub-argumentation frameworks:

Definition 3 [25] Let $A F=(A, R)$ be an argumentation framework, $S$ a subset of $A$. The restriction of $A F$ to $S$, denoted by $\left.A F\right|_{S}$, is the sub-argumentation framework $(S, R \cap(S \times$ $S))$.

Remark 2 For any nonempty subset $S$ of $A$, by the relation $R$ we can divide the set $A$ into three disjoint parts: $S, R^{+}(S)$ and $A \backslash\left(S \cup R^{+}(S)\right)$. In our discussion on division of $A F$ in Section 6, the sub-argumentation $\left.A F\right|_{A \backslash\left(S \cup R^{+}(S)\right)}$ plays an important role. In order to emphasize its importance, we call it the remaining sub-argumentation wrt $S$, or remaining sub-argumentation for short.

\section{The matrix representation}

Let $A F=(A, R)$ be an argumentation framework. It is convenient to put $A=\{1,2, \ldots, n\}$ whenever the cardinality $A$ is large. Furthermore, we usually give the set $A$ a permutation, for example $\left(i_{1}, i_{2}, \ldots, i_{n}\right)$, when dealing with the objects of $A F$ practically.

Definition 4 Let $A F=(A, R)$ be an argumentation framework with $A=\{1,2, \ldots, n\}$. The matrix of $A F$ corresponding to the permutation $\left(i_{1}, i_{2}, \ldots, i_{n}\right)$ of $A$, denoted by $M\left(i_{1}, i_{2}, \ldots, i_{n}\right)^{1}$, is a boolean matrix of order $n$, its elements are determined by the following rules:

(1) $a_{s, t}=1$ iff $\left(i_{s}, i_{t}\right) \in R$

(2) $a_{s, t}=0$ iff $\left(i_{s}, i_{t}\right) \notin R$

For any matrix $M$, the $i$-th row is sometimes called the $i$-th row vector and denoted by $M_{i, *}$, the $j$-th column is sometimes called $j$-th column vector and denoted by $M_{*, j}$. We will adopt this notation in the subsequent discussion.

Example 1 Given $A F=(A, R)$ with $A=\{1,2,3\}$ and $R=\{(1,2),(2,3),(3,1)\}$, represented by the following graph:

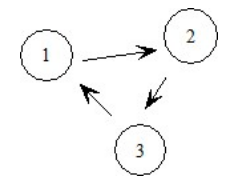

\footnotetext{
${ }^{1}$ strictly speaking, it should be denoted by $M_{A F}\left(i_{1}, i_{2}, \ldots, i_{n}\right)$
} 
For the natural permutation $\left(i_{1}, i_{2}, i_{3}\right)=(1,2,3)$, we have the corresponding relation as follows:

$$
\begin{aligned}
\left(i_{1}, i_{2}, i_{3}\right)=(1,2,3) \quad & 1 \rightarrow 2 \Leftrightarrow i_{1} \rightarrow i_{2} \Leftrightarrow a_{12}=1 ; \\
& 2 \rightarrow 3 \Leftrightarrow i_{2} \rightarrow i_{3} \Leftrightarrow a_{23}=1 ; \\
& 3 \rightarrow 1 \Leftrightarrow i_{3} \rightarrow i_{1} \Leftrightarrow a_{31}=1 . \\
& \text { Other } \mathrm{a}_{\mathrm{ij}}=0 .
\end{aligned}
$$

And thus, the matrix of AF corresponding to $(1,2,3)$ is:

$$
\begin{aligned}
& M(1,2,3)=M\left(i_{1}, i_{2}, i_{3}\right) \\
& =\left(\begin{array}{lll}
a_{11} & a_{12} & a_{13} \\
a_{21} & a_{22} & a_{23} \\
a_{31} & a_{32} & a_{33}
\end{array}\right)=\left(\begin{array}{lll}
0 & 1 & 0 \\
0 & 0 & 1 \\
1 & 0 & 0
\end{array}\right)
\end{aligned}
$$

\begin{tabular}{c|c|c|c} 
& 1 & 2 & 3 \\
\hline 1 & 0 & 1 & 0 \\
\hline 2 & 0 & 0 & 1 \\
\hline 3 & 1 & 0 & 0 \\
\hline
\end{tabular}

If we consider the permutation $\left(i_{1}, i_{2}, i_{3}\right)=(1,3,2)$, the corresponding relation is as follows:

$$
\begin{aligned}
\left(i_{1}, i_{2}, i_{3}\right)=(2,1,3) \quad & 2 \rightarrow 3 \Leftrightarrow i_{1} \rightarrow i_{3} \Leftrightarrow a_{13}=1 ; \\
& 3 \rightarrow 1 \Leftrightarrow i_{3} \rightarrow i_{2} \Leftrightarrow a_{32}=1 . \\
& \text { Other } \mathrm{a}_{\mathrm{ij}}=0 .
\end{aligned}
$$

And thus, the matrix of AF corresponding to $(1,3,2)$ is:

$$
\begin{aligned}
& M(2,1,3)=M\left(i_{1}, i_{2}, i_{3}\right) \\
& =\left(\begin{array}{lll}
a_{11} & a_{12} & a_{13} \\
a_{21} & a_{22} & a_{23} \\
a_{31} & a_{32} & a_{33}
\end{array}\right)=\left(\begin{array}{lll}
0 & 0 & 1 \\
1 & 0 & 0 \\
0 & 1 & 0
\end{array}\right)
\end{aligned}
$$

\begin{tabular}{c|c|c|c|} 
& 2 & 1 & 3 \\
\hline 2 & 0 & 0 & 1 \\
\hline 1 & 1 & 0 & 0 \\
\hline 3 & 0 & 1 & 0 \\
\hline
\end{tabular}

In matrix $M\left(i_{1}, i_{2}, \ldots, i_{n}\right)$, the value of $a_{s, t}$ represents the attack relation of $i_{s}$ to $i_{t}$. So, the argumentation framework $A F$ has many different matrix representations, which depend on the different permutations of $A$. Although $A F$ has many different matrix representations, all these matrices have the same role in representing $A F$. We usually employ the matrix $M(1,2, \ldots, n)$ to represent $A F=(A, R)$, and denote it by $M(A F)$.

In Example1, the permutation $(1,3,2)$ can be obtained by exchanging 2 with 3 from $(1,2,3)$. Correspondingly, by exchanging row 2 with row 3 , and column 2 with column 3 in matrix $M(1,2,3)$ we can get the matrix $M(1,3,2)$. These exchanges can be formalized with an operation on matrices called dual interchange.

Definition 5 Let $A F=(A, R)$ be an argumentation framework with $A=\{1,2, \ldots, n\}$ and $M\left(i_{1}, \ldots, i_{k}, \ldots, i_{l}, \ldots, i_{n}\right)$ be the matrix of $A F$ corresponding to the permutation $\left(i_{1}, \ldots\right.$, $\left.i_{k}, \ldots, i_{l}, \ldots, i_{n}\right)$ of $A$. A dual interchange on the matrix $M\left(i_{1}, \ldots, i_{k}, \ldots, i_{l}, \ldots, i_{n}\right)$ between $k$ 
and $l$, denoted by $k \rightleftharpoons l$, consists of two exchanges: exchanging $k$-th row and $l$-th row; exchanging $k$-th column and $l$-th column.

Lemma 1 Let $A F=(A, R)$ be an argumentation framework with $A=\{1,2, \ldots, n\}$, the dual interchange $i_{k} \rightleftharpoons i_{l}$ turns the matrix $M\left(i_{1}, \ldots, i_{k}, \ldots, i_{l}, \ldots, i_{n}\right)$ into the matrix $M\left(i_{1}, \ldots, i_{l}, \ldots, i_{k}, \ldots, i_{n}\right)$.

Remark 3 Note that the dual interchange $k \rightleftharpoons l$ also turns the matrix $M\left(i_{1}, \ldots, i_{l}, \ldots, i_{k}, \ldots, i_{n}\right)$ into the matrix $M\left(i_{1}, \ldots, i_{k}, \ldots, i_{l}, \ldots, i_{n}\right)$. So, for any two matrices of AF corresponding to different permutations of $A$ we can turn one matrix into another by a sequence of dual interchanges. In this sense, we may call them to be equivalent matrix representations of $A F$.

Example 1 (cont'd): By the dual interchange $1 \rightleftharpoons 2$, we can turn the matrix $M(1,2,3)$ into the matrix $M(2,1,3)$.

$$
\left(\begin{array}{lll}
0 & 1 & 0 \\
0 & 0 & 1 \\
1 & 0 & 0
\end{array}\right) \underline{1 \rightleftharpoons 2}\left(\begin{array}{lll}
0 & 0 & 1 \\
1 & 0 & 0 \\
0 & 1 & 0
\end{array}\right)
$$

\section{Characterizing the extensions of an $A F$}

By definition 4, the matrix $M(A F)$ contains all the information of $A F$. For the interaction of any two arguments, the value of the corresponding element in the matrix gives its representation. But for the extensions of $A F$ (such as admissible, complete, stable) they have no obvious feature in the matrix $M(A F)$, i.e., we can not decide whether a subset of $A$ is a stable (admissible, complete) extension from $M(A F)$ directly. In this section, we mainly focus on finding the characterization of various extensions in the matrix $M(A F)$ of $A F$. The idea is to establish the relation between the extensions (viewed as subsets) of $A F$ and the subblocks of $M(A F)$.

\subsection{Characterizing the conflict-free subsets}

Note that a self-conflicting argument is not contained in any conflict-free subset (and thus not contained in any admissible extension). So we may omit the self-conflicting arguments to avoid unnecessary trouble when considering the conflict-free subsets and basic extensions.

The first basic requirement for any extension is the conflict-free principle, i.e., if an argument $i$ attacks another argument $j$ then they can not be included together in an extension. So, we will discuss the matrix condition which insures that a subset of an argumentation framework is conflict-free. 
Definition 6 Let $A F=(A, R)$ be an argumentation framework with $A=\{1,2, \ldots, n\}$, and $S=\left\{i_{1}, i_{2}, \ldots, i_{k}\right\} \subseteq A$. The principal $k \times k$ subblock

$$
M_{i_{1}, i_{2}, \ldots, i_{k}}^{i_{1}, i_{2}, \ldots, i_{k}}=\left(\begin{array}{cccccc}
a_{i_{1}, i_{1}} & a_{i_{1}, i_{2}} & \cdot & . & \cdot & a_{i_{1}, i_{k}} \\
a_{i_{2}, i_{1}} & a_{i_{2}, i_{2}} & \cdot & \cdot & \cdot & a_{i_{2}, i_{k}} \\
\cdot & \cdot & \cdot & \cdot & \cdot & \cdot \\
a_{i_{k}, i_{1}} & a_{i_{k}, i_{2}} & \cdot & \cdot & \cdot & a_{i_{k}, i_{k}}
\end{array}\right)
$$

of $M(A F)$ is called the cf-subblock of $S$, and denoted by $M^{c f}(S)$ for short.

Theorem 1 Given $A F=(A, R)$ with $A=\{1,2, \ldots, n\}, S=\left\{i_{1}, i_{2}, \ldots, i_{k}\right\} \subseteq A$ is conflictfree iff the cf-subblock $M^{\text {cf }}(S)$ is zero.

Example 2 Let $(A, R)$ be an argumentation framework with $A=\{1,2,3\}$ and $R=$ $\{(1,3),(2,3),(3,1)\}$, then the matrix and graph of $A F$ are as follows:

$$
M(A F)=\left(\begin{array}{ccc}
0 & 0 & 1 \\
0 & 0 & 1 \\
1 & 0 & 0
\end{array}\right)
$$

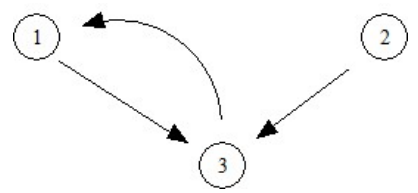

We have $M^{c f}(\{1,2\})=\left(\begin{array}{ll}0 & 0 \\ 0 & 0\end{array}\right), M^{c f}(\{1,3\})=\left(\begin{array}{cc}0 & 1 \\ 1 & 0\end{array}\right)$, and $M^{c f}(\{2,3\})=$ $\left(\begin{array}{ll}0 & 1 \\ 0 & 0\end{array}\right)$. By Theorem 1, $\{1,2\}$ is conflict free, $\{1,3\}$ and $\{2,3\}$ are not.

\subsection{Characterizing the stable extensions}

As shown in Section2, a subset $S$ of $A$ is stable if and only if $R^{+}(S)=A \backslash S$. From the viewpoint of set theory, every stable extension $S$ separates $A$ into two disjoint parts: $S$ and $A \backslash S=R^{+}(S)$. So, except for the conflict-freeness of $S$, we only need to concentrate on whether the arguments in $A \backslash S$ are attacked by $S$. We need not to concern about the attacked situation of arguments in $S$ and the relationship between arguments in $A \backslash S$. In this sense, stable semantics is bipartite and one-way. This suggests the following definition:

Definition 7 Let $A F=(A, R)$ be an argumentation framework with $A=\{1,2, \ldots, n\}$, $S=\left\{i_{1}, i_{2}, \ldots, i_{k}\right\} \subseteq A$ and $A \backslash S=\left\{j_{1}, j_{2}, \ldots, j_{h}\right\}$. The $k \times h$ subblock

$$
M_{j_{1}, j_{2}, \ldots, j_{h}}^{i_{1}, i_{2}, \ldots, i_{k}}=\left(\begin{array}{cccccc}
a_{i_{1}, j_{1}} & a_{i_{1}, j_{2}} & \cdot & \cdot & \cdot & a_{i_{1}, j_{h}} \\
a_{i_{2}, j_{1}} & a_{i_{2}, j_{2}} & \cdot & \cdot & \cdot & a_{i_{2}, j_{h}} \\
\cdot & \cdot & \cdot & \cdot & \cdot & \cdot \\
a_{i_{k}, j_{1}} & a_{i_{k}, j_{2}} & \cdot & \cdot & \cdot & a_{i_{k}, j_{h}}
\end{array}\right)
$$

of $M(A F)$ is called s-subblock of $S$ and denoted by $M^{s}(S)$ for short. 
In other words, we take the rows $i_{1}, i_{2}, \ldots, i_{k}$ and the columns $j_{1}, j_{2}, \ldots, j_{h}$ in the matrix $M(A F)$.

Theorem 2 Given an argumentation framework $A F=(A, R)$ with $A=\{1,2, \ldots, n\}$, the conflict-free subset $S=\left\{i_{1}, i_{2}, \ldots, i_{k}\right\} \subseteq A$ is a stable extension iff each column vector of the s-subblock $M^{s}(S)=M_{j_{1}, j_{2}, \ldots, j_{h}}^{i_{1}, i_{2}, \ldots, i_{k}}$ of $M(\bar{A} F)$ is non-zero, where $\left(j_{1}, j_{2}, \ldots, j_{h}\right)$ is a permutation of $A \backslash S$.

Example 2 (cont'd):

$$
M(A F)=\left(\begin{array}{lll}
0 & 0 & 1 \\
0 & 0 & 1 \\
1 & 0 & 0
\end{array}\right)
$$

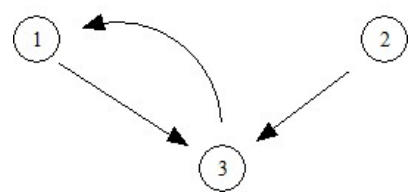

We consider the conflict-free subsets $\{1\}$ and $\{1,2\}$. Since the first column vector of $M^{s}(\{1\})=\left(\begin{array}{ll}0 & 1\end{array}\right)$ is zero and the only column vector of $M^{s}(\{1,2\})=\left(\begin{array}{l}1 \\ 1\end{array}\right)$ is nonzero, we claim that $\{1,2\}$ is a stable extension of $A F$ but $\{1\}$ is not, according to Theorem 2.

\subsection{Characterizing the admissible subsets}

As shown in Section2, a subset $S$ of $A$ is admissible if and only if $R^{-}(S) \subseteq R^{+}(S) \subseteq A \backslash S$. So there may be arguments in $A \backslash S$ which are not attacked by $S$. Such arguments should not attack $S$. So, we have to deal with the arguments in $A \backslash S$ in different ways: arguments attacked by $S$, arguments not attacked by $S$ (should not attack $S$ if $S$ is admissible).

Definition 8 Let $A F=(A, R)$ be an argumentation framework with $A=\{1,2, \ldots, n\}$, $S=\left\{i_{1}, i_{2}, \ldots, i_{k}\right\} \subseteq A$ and $A \backslash S=\left\{j_{1}, j_{2}, \ldots, j_{h}\right\}$. The $h \times k$ subblock

$$
M_{i_{1}, i_{2}, \ldots, i_{k}}^{j_{1}, j_{2}, \ldots, j_{h}}=\left(\begin{array}{cccccc}
a_{j_{1}, i_{1}} & a_{j_{1}, i_{2}} & \cdot & \cdot & \cdot & a_{j_{1}, i_{k}} \\
a_{j_{2}, i_{1}} & a_{j_{2}, i_{2}} & \cdot & \cdot & \cdot & a_{j_{2}, i_{k}} \\
\cdot & \cdot & \cdot & \cdot & \cdot & \cdot \\
a_{j_{h}, i_{1}} & a_{j_{h}, i_{2}} & \cdot & \cdot & \cdot & a_{j_{h}, i_{k}}
\end{array}\right)
$$

of $M(A F)$ is called a-subblock of $S$ and denoted by $M^{a}(S)$.

In other words, we take the rows $j_{1}, j_{2}, \ldots, j_{h}$ and the columns $i_{1}, i_{2}, \ldots, i_{k}$ in the matrix $M(A F)$.

From the viewpoint of set theory, every admissible set $S$ separates $A$ into three disjoint parts: $S, R^{+}(S)$ and $A \backslash\left(S \cup R^{+}(S)\right)$. Except for the conflict-freeness of $S\left(R^{+}(S) \subseteq A \backslash S\right)$, we only need to consider whether $S$ is attacked by the arguments in $A \backslash\left(S \cup R^{+}(S)\right)$. We need not to concern about if arguments in $S$ are attacked by $R^{+}(S)$ and the relationship between arguments in $A \backslash\left(S \cup R^{+}(S)\right)$ as well as in $R^{+}(S)$. In this sense, admissible semantics is tripartite and one-way. 
Theorem 3 Given an argumentation framework $A F=(A, R)$ with $A=\{1,2, \ldots, n\}$, then the conflict-free subset $S=\left\{i_{1}, i_{2}, \ldots, i_{k}\right\} \subseteq A$ is admissible iff any column vector of the $s$-subblock $M^{s}(S)$ corresponding to a non-zero row vector of the a-subblock $M^{a}(S)$ is nonzero, where $\left(j_{1}, j_{2}, \ldots, j_{h}\right)$ is a permutation of $A \backslash S$.

\section{Example 2 (cont'd):}

$$
M(A F)=\left(\begin{array}{lll}
0 & 0 & 1 \\
0 & 0 & 1 \\
1 & 0 & 0
\end{array}\right)
$$

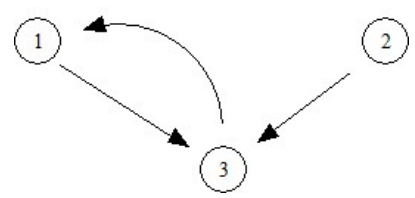

Next, we consider the conflict-free subsets: $\{1\}$ and $\{3\}$.

Since $M^{s}(\{1\})=\left(\begin{array}{ll}0 & 1\end{array}\right)$ and $M^{a}(\{1\})=\left(\begin{array}{l}0 \\ 1\end{array}\right)$, the second column vector of $M^{s}(\{1\})$ corresponding to the second non-zero row vector of $M^{a}(\{1\})$ is non-zero, we claim that $\{1\}$ is admissible in AF by Theorem 3.

But, from $M^{s}(\{3\})=\left(\begin{array}{ll}1 & 0\end{array}\right)$ and $M^{a}(\{3\})=\left(\begin{array}{l}1 \\ 1\end{array}\right)$ we know that the second column vector of $M^{s}(\{3\})$ corresponding to the second non-zero row vector of $M^{a}(\{3\})$ is zero. So, $\{3\}$ is not admissible in AF according to Theorem 3.

Remark 4 Note that the condition "each column vector of the s-subblock $M^{s}(S)$ is nonzero" in Theorem 2 is stronger than that "any column vector of the s-subblock $M^{s}(S)$ corresponding to a non-zero row vector of the a-subblock $M^{a}(S)$ is non-zero", and this is exactly the matrix expression of the fact that any stable extension must be admissible.

\subsection{Characterizing the complete extensions}

From the viewpoint of set theory, every complete extension $S$ separates $A$ into three disjoint parts: $S, R^{+}(S)$ and $A \backslash\left(S \cup R^{+}(S)\right)$. Except for the conflict freeness of $S$, we need not only to consider whether $S$ is attacked by the arguments in $A \backslash\left(S \cup R^{+}(S)\right)$, but also to see if every argument in $A \backslash\left(S \cup R^{+}(S)\right)$ is attacked by some others in $A \backslash\left(S \cup R^{+}(S)\right)$. We need not to concern about if arguments in $S$ are attacked by $R^{+}(S)$ and the relationship between arguments in $R^{+}(S)$. In this sense, complete semantics is tripartite and two-way.

Definition 9 Let $A F=(A, R)$ be an argumentation framework with $A=\{1,2, \ldots, n\}$, $S=\left\{i_{1}, i_{2}, \ldots, i_{k}\right\} \subseteq A$ and $A \backslash S=\left\{j_{1}, j_{2}, \ldots, j_{h}\right\}$. The $h \times h$ subblock

$$
M_{j_{1}, j_{2}, \ldots, j_{h}}^{j_{1}, j_{2}, \ldots, j_{h}}=\left(\begin{array}{cccccc}
a_{j_{1}, j_{1}} & a_{j_{1}, j_{2}} & \cdot & \cdot & \cdot & a_{j_{1}, j_{h}} \\
a_{j_{2}, j_{1}} & a_{j_{2}, j_{2}} & \cdot & \cdot & \cdot & a_{j_{2}, j_{h}} \\
\cdot & \cdot & \cdot & \cdot & \cdot & \cdot \\
a_{j_{h}, j_{1}} & a_{j_{h}, j_{2}} & \cdot & \cdot & \cdot & a_{j_{h}, j_{h}}
\end{array}\right)
$$

of $M(A F)$ is called c-subblock of $S$ and denoted by $M^{c}(S)$ for short. 
In other words, we take the rows $j_{1}, j_{2}, \ldots, j_{h}$ and the columns $j_{1}, j_{2}, \ldots, j_{h}$ in the matrix $M(A F)$.

Theorem 4 Given an argumentation framework $A F=(A, R)$ with $A=\{1,2, \ldots, n\}$, the admissible set $S=\left\{i_{1}, i_{2}, \ldots, i_{k}\right\} \subseteq A$ is complete iff the column vector $M_{*, p}^{c}$ of the $c$ subblock $M^{c}(S)$ of $S$ corresponding to the zero column vector $M_{*, p}^{s}$ of the s-subblock $M^{s}(S)$ of $S$ is non-zero, and there is at least one non-zero element $a_{j_{q}, j_{p}}$ of $M_{*, p}^{c}$ such that the corresponding column vector $M_{*, q}^{s}$ of the s-block $M^{s}(S)$ of $S$ is zero, where $\left\{j_{1}, j_{2}, \ldots, j_{h}\right\}=$ $A \backslash S$ and $1 \leq q, p \leq h$.

Example 3 Let $A F=(A, R)$ be an argumentation framework with $A=\{1,2,3,4,5\}$ and $R=\{(2,5),(3,4),(4,3),(5,1),(5,3)\}$. The matrix and graph of $A F$ are as follows:

$$
M(A F)=\left(\begin{array}{ccccc}
0 & 0 & 0 & 0 & 0 \\
0 & 0 & 0 & 0 & 1 \\
0 & 0 & 0 & 1 & 0 \\
0 & 0 & 1 & 0 & 0 \\
1 & 0 & 1 & 0 & 0
\end{array}\right)
$$

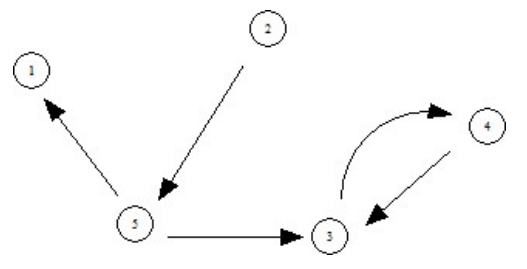

By Theorem 3, it is easy to verify that $S=\{1,2\}$ is admissible. Next, we will denote $i_{1}=1, i_{2}=2, j_{1}=3, j_{2}=4, j_{3}=5$ and check whether $S$ is complete.

Note that $M^{s}(\{1,2\})=\left(\begin{array}{lll}0 & 0 & 0 \\ 0 & 0 & 1\end{array}\right)$ has two zero column vectors $M_{*, 1}^{s}=\left(\begin{array}{l}0 \\ 0\end{array}\right)$ and $M_{*, 2}^{s}=\left(\begin{array}{l}0 \\ 0\end{array}\right)$. Also, their corresponding column vectors in $M^{c}(\{1,2\})=\left(\begin{array}{lll}0 & 1 & 0 \\ 1 & 0 & 0 \\ 1 & 0 & 0\end{array}\right)$ are $M_{*, 1}^{c}=\left(\begin{array}{l}0 \\ 1 \\ 1\end{array}\right)$ and $M_{*, 2}^{c}=\left(\begin{array}{l}1 \\ 0 \\ 0\end{array}\right)$ respectively, which are all nonzero. For $a_{j_{2} j_{1}}=a_{43}=1$ in $M_{*, 1}^{c}$, the corresponding column vector $M_{*, 2}^{s}$ in $M^{s}(\{1,2\})$ is zero. For $a_{j_{1} j_{2}}=a_{34}=1$ in $M_{*, 2}^{c}$, the corresponding column vector $M_{*, 1}^{s}$ in $M^{s}(\{1,2\})$ is also zero. According to Theorem 4, we claim that $\{1,2\}$ is a complete extension of $A F$.

By now, we can determine the extensions of three basic semantics through checking the sub-blocks of the matrix of $A F$. Note that the rules in each theorem are obtained directly from the corresponding definition of semantics. So, there is no more advantage than judging by definitions. In the next subsection, we will improve the rules to achieve some unified form by which one can determine different extensions easily.

\subsection{The standard forms of the matrix $M(A F)$}

In linear algebra, one can reduce the matrix of a system of linear equations into row echelon form by row transformations. Then, it is easy to find the solution of the system of linear 
equations from this row echelon form. Similarly, we will use dual interchange to reduce the matrix of $A F$ into some kind of standard form, by which the extensions discussed above can be easily determined. In the sequel, two standard forms are introduced with respect to different semantics. One is based on a partition of the matrix of order two, and the other one uses a partition of order three.

From the definitions of s-subblock, a-subblock and c-subblock, we can see that the matrix of $A F$ is exactly divided into four parts: the entries representing relations between the arguments in $S$, the entries representing the relations between arguments in $S$ and arguments in $R^{+}(S), \ldots$. The problem is that these classes of entries are distributed in the matrix disorderly. So, we need to rearrange them in a proper way such that they appear clearly and can be easily treated.

Theorem 5 Let $A F=(A, R)$ be an argumentation framework with $A=\{1,2, \ldots, n\}$, $S=\left\{i_{1}, i_{2}, \ldots, i_{k}\right\} \subseteq A$. Then, by a sequence of dual interchanges we can turn $M(A F)$ into the matrix $M\left(i_{1}, i_{2}, \ldots, i_{k}, j_{1}, j_{2}, \ldots, j_{h}\right)$, which has the following form

$$
\left(\begin{array}{cc}
M^{c f}(S) & M^{s}(S) \\
M^{a}(S) & M^{c}(S)
\end{array}\right)
$$

where $\left\{j_{1}, j_{2}, \ldots, j_{h}\right\}=A \backslash S$, and $M^{c f}(S), M^{s}(S), M^{a}(S), M^{c}(S)$ are the cf- subblock, s-subblock, a-subblock, c-subblock of $S$ of the matrix $M(A F)$ respectively.

Corollary 1 Given $A F=(A, R)$ with $A=\{1,2, \ldots, n\}$, and $S=\left\{i_{1}, i_{2}, \ldots, i_{k}\right\}, A \backslash S=$ $\left\{j_{1}, \ldots, j_{h}\right\}$. Let $M\left(i_{1}, i_{2}, \ldots, i_{k}, j_{1}, \ldots, j_{h}\right)$ be the matrix of $A F$ corresponding to the permutation $\left(i_{1}, i_{2}, \ldots, i_{k}, j_{1}, \ldots, j_{h}\right)$, we have $S$ conflict-free iff

$$
M\left(i_{1}, i_{2}, \ldots, i_{k}, j_{1}, \ldots, j_{h}\right)=\left(\begin{array}{cc}
0 & M^{s}(S) \\
M^{a}(S) & M^{c}(S)
\end{array}\right)
$$

Proof: It follows directly from Theorem 1.

Corollary 2 Given $A F=(A, R)$ with $A=\{1,2, \ldots, n\}$, and $S=\left\{i_{1}, i_{2}, \ldots, i_{k}\right\}, A \backslash S=$ $\left\{j_{1}, \ldots, j_{h}\right\}$. Let $M\left(i_{1}, i_{2}, \ldots, i_{k}, j_{1}, \ldots, j_{h}\right)$ be the matrix of $A F$ corresponding to the permutation $\left(i_{1}, i_{2}, \ldots, i_{k}, j_{1}, \ldots, j_{h}\right)$, we have $S$ stable iff the $c f$-subblock $M^{c f}(S)=0$ and every column vector of the s-subblock $M^{s}(S)$ is not non-zero.

Proof: It follows directly from Theorem 2.

Example 4 Let $A F=(A, R)$ be an argumentation framework with $A=\{1,2,3,4\}$ and $R=\{(1,3),(2,3),(3,2),(3,4),(4,1)\}$, then the matrix and graph of $A F$ are as follows: 


$$
M(A F)=\left(\begin{array}{cccc}
0 & 0 & 1 & 0 \\
0 & 0 & 1 & 0 \\
0 & 1 & 0 & 1 \\
1 & 0 & 0 & 0
\end{array}\right)
$$

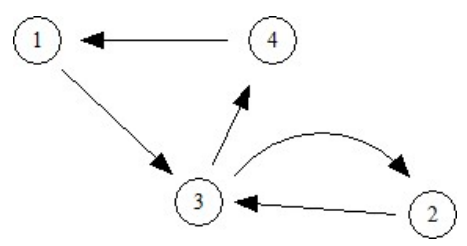

Obviously, $S=\{2,4\}$ is a conflict-free subset of $A F$. By the dual interchange $1 \rightleftarrows 4$, $M(A F)$ can be turned into the following matrix:

$$
M(4,2,3,1)=\left(\begin{array}{llll}
0 & 0 & 0 & 1 \\
0 & 0 & 1 & 0 \\
1 & 1 & 0 & 0 \\
0 & 0 & 1 & 0
\end{array}\right) .
$$

Since $M^{s}(S)=\left(\begin{array}{ll}0 & 1 \\ 1 & 0\end{array}\right),\{2,4\}$ is a stable extension of AF by Corollary 2.

We have obtained a partition of the matrix of order two, composed by four kind of sub-blocks, from which we can determine the conflict-free status of $S$ and the stable status of $S$. However, there is no more new information to deal with admissible and complete extensions. In fact, for any conflict free subset $S$ we can divide $A$ into three disjoint subsets: $S, R^{+}(S)$ and $A \backslash\left(S \cup R^{+}(S)\right)$. Motivated by this idea, we can transform the partition of order two into a new partition of order three. It can be thought as a standard form of the matrix $M(A F)$, by which we can determine the admissible and complete extensions (because we only need to check one or two sub-blocks of less order to see whether it is zero).

Theorem 6 Let $A F=(A, R)$ be an argumentation framework with $A=\{1,2, \ldots, n\}$, $S=\left\{i_{1}, i_{2}, \ldots, i_{k}\right\} \subseteq A$ be a conflict-free subset. Then, by a sequence of dual interchanges we can turn $M(A F)$ into the following matrix

$$
M\left(i_{1}, i_{2}, \ldots, i_{k}, j_{t_{1}}, \ldots, j_{t_{q}}, j_{s_{1}}, \ldots, j_{s_{l}}\right)=\left(\begin{array}{ccc}
0_{k, k} & 0_{k, q} & S_{k, l} \\
A_{q, k} & C_{q, q} & E_{q, l} \\
F_{l, k} & G_{l, q} & H_{l, l}
\end{array}\right)=\left(\begin{array}{cc}
0_{k, k} & M^{s}(S) \\
M^{a}(S) & M^{c}(S)
\end{array}\right)
$$

where $A \backslash S=\left\{j_{t_{1}}, \ldots, j_{t_{q}}, j_{s_{1}}, \ldots, j_{s_{l}}\right\}, k+q+l=k+h=n$, and each column vector of $S_{k, l}$ is non-zero.

Corollary 3 Given $A F=(A, R)$ with $A=\{1,2, \ldots, n\}$, and $S=\left\{i_{1}, i_{2}, \ldots, i_{k}\right\}, A \backslash S=$ $\left\{j_{t_{1}}, \ldots, j_{t_{q}}, j_{s_{1}}, \ldots, j_{s_{l}}\right\}$. Let $M\left(i_{1}, i_{2}, \ldots, i_{k}, j_{t_{1}}, \ldots, j_{t_{q}}, j_{s_{1}}, \ldots, j_{s_{l}}\right)$ be the matrix of AF corresponding to the permutation $\left(i_{1}, i_{2}, \ldots, i_{k}, j_{t_{1}}, \ldots, j_{t_{q}}, j_{s_{1}}, \ldots, j_{s_{l}}\right)$ as in Theorem 6 , then $S$ is an admissible extension iff $A_{q, k}=0$.

Proof: It follows from Theorem 3 and Theorem 6 . 
Remark 5 Note that Corollary 3 states that a conflict-free subset $S$ is admissible iff it does not conflict with $A \backslash\left(S \cup R^{+}(S)\right)$.

\section{Example 4 (cont'd):}

$$
M(A F)=\left(\begin{array}{cccc}
0 & 0 & 1 & 0 \\
0 & 0 & 1 & 0 \\
0 & 1 & 0 & 1 \\
1 & 0 & 0 & 0
\end{array}\right)
$$

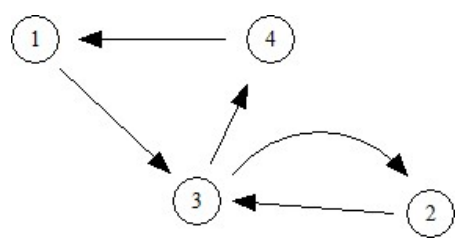

Obviously, $S=\{2\}$ is a conflict-free subset of AF. By taking the sequence of dual interchanges $1 \rightleftarrows 2$ and $3 \rightleftarrows 4, M(A F)$ can be turned into the following matrix:

$$
M(2,1,4,3)=\left(\begin{array}{llll}
0 & 0 & 0 & 1 \\
0 & 0 & 0 & 1 \\
0 & 1 & 0 & 0 \\
1 & 0 & 1 & 0
\end{array}\right) .
$$

Note that here $i_{1}=2, j_{t_{1}}=1, j_{t_{2}}=4$ and $j_{s_{1}}=3$ with $k=1, q=2, l=1$. Since $S_{k, l}=S_{1,1}=(1), A_{q, k}=A_{2,1}=\left(\begin{array}{l}0 \\ 0\end{array}\right)$, we claim that $\{2\}$ is an admissible extension of AF according to Corollary 3.

Corollary 4 Given $A F=(A, R)$ with $A=\{1,2, \ldots, n\}$, and $S=\left\{i_{1}, i_{2}, \ldots, i_{k}\right\}, A \backslash S=$ $\left\{j_{t_{1}}, \ldots, j_{t_{q}}, j_{s_{1}}, \ldots, j_{s_{l}}\right\}$. Let $M\left(i_{1}, i_{2}, \ldots, i_{k}, j_{t_{1}}, \ldots, j_{t_{q}}, j_{s_{1}}, \ldots, j_{s_{l}}\right)$ be the matrix of AF corresponding to the permutation $\left(i_{1}, i_{2}, \ldots, i_{k}, j_{t_{1}}, \ldots, j_{t_{q}}, j_{s_{1}}, \ldots, j_{s_{l}}\right)$ as in above Theorem 6 , then $S$ is a complete extension iff $A_{q, k}=0$ and each column vector of $C_{q, q}$ is not zero.

Proof: It follows from Theorem 4 and Theorem 6 .

Remark 6 Corollary 4 states that a conflict-free subset $S$ is complete iff it does not conflict with $A \backslash\left(S \cup R^{+}(S)\right)$ and there is no initial argument in the sub-argumentation framework $\left.A F\right|_{A \backslash\left(S \cup R^{+}(S)\right)} \cdot$

\section{Example 3 (cont'd):}

$$
M(A F)=\left(\begin{array}{ccccc}
0 & 0 & 0 & 0 & 0 \\
0 & 0 & 0 & 0 & 1 \\
0 & 0 & 0 & 1 & 0 \\
0 & 0 & 1 & 0 & 0 \\
1 & 0 & 1 & 0 & 0
\end{array}\right)
$$

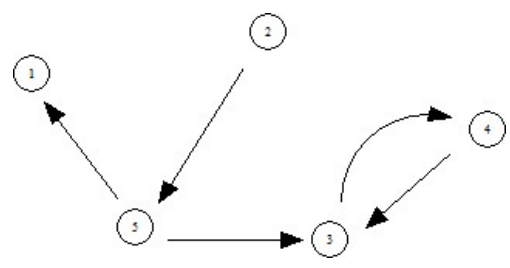

Obviously, $S=\{1,2\}$ is a conflict-free subset of $A F$. Note that, $M(A F)$ has already the normal form we need for $S$. Here, $i_{1}=1, i_{2}=2, j_{t_{1}}=3, j_{t_{2}}=4$ and $j_{s_{1}}=5$ with $k=2, q=2, l=1$. Because $S_{k, l}=S_{2,1}=\left(\begin{array}{l}0 \\ 1\end{array}\right), A_{q, k}=A_{2,2}=\left(\begin{array}{ll}0 & 0 \\ 0 & 0\end{array}\right)$, we deduce that $S$ is admissible by Corollary 3. 
Furthermore, we have $C_{q, q}=C_{2,2}=\left(\begin{array}{ll}0 & 1 \\ 1 & 0\end{array}\right)$, and thus $\{1,2\}$ is a complete extension of AF according to Corollary 4.

\section{Matrix reduction based on the contraction of $A F$}

In Section 4, we proposed to characterize the stable (admissible, complete) extensions of an argumentation framework by dividing $A$ into two or three parts, and then considering the interaction between these different parts. This motivates us to consider the idea of contracting one part of an argumentation framework (namely a conflict-free subset) into a single argument by drawing up some rules. By this way, the argumentation framework will be compressed into another argumentation framework with less arguments. And thus, we can reduce the matrix into another matrix of less order which plays the same role for our purpose. Note that it is trivial to contract a conflict-free subset with only one argument. So, only the conflict-free subsets with more than one argument are considered in this section.

Since there are many conflict-free subsets in an argumentation framework, some of them may intersect and some may not. So we discuss three types of reduction wrt conflict-free subsets. In order to describe the reduction process, we need to define a special addition of rows (respectively columns) of a matrix as follows:

Definition 10 Let $M(A F)$ be the matrix of an argumentation framework. Adding two rows of the matrix $M(A F)$ consists in adding the elements in the same position of the rows with the rules " $0+0=0,0+1=1,1+1=1 "$. The addition of columns is as the same.

\subsection{The first type of reduction}

Let $A F=(A, R)$ be an argumentation framework with $A=\{1,2, \ldots, n\}$ and $M(A F)$ the matrix of $A F$. For a subset $S=\left\{i_{1}, i_{2}, \ldots, i_{k}\right\}$ of $A F$, what we think about is to contract the sub-block $M^{c f}(S)$ into a single entry in the matrix and this entry shares the same status as $M^{c f}(S)$ wrt semantics.

Now, let $S=\left\{i_{1}, i_{2}, \ldots, i_{k}\right\}$ be a conflict-free subset of $A F$. We reduce the matrix $M(A F)$ into another matrix $M_{S}^{r 1}(A F)$ of order $n-k+1$ by the following rules:

Let $1 \leq t \leq k$,

1. adding every row $i_{s}$ to the row $i_{t}$,

2. adding every column $i_{s}$ to the column $i_{t}$, then

3. deleting every row $i_{s}$ and column $i_{s}$,

where $1 \leq s \leq k$ and $s \neq t$.

The matrix $M_{S}^{r 1}(A F)$ is called the reduced matrix wrt $r 1$ and the conflict-free subset $S$, 
or the reduced matrix wrt $S$ for short.

Corresponding to the above process for matrix, we can reduce the original argumentation framework into a new one with $n-k+1$ arguments. For that purpose, the following rules are applied.

Let $A \backslash S=\left\{j_{1}, j_{2}, \ldots, j_{h}\right\}$ and $1 \leq t \leq k$,

1. adding $\left(i_{t}, j_{q}\right)$ to $R$ if $\left(i_{s}, j_{q}\right) \in R$,

2. adding $\left(j_{q}, i_{t}\right)$ to $R$ if $\left(j_{q}, i_{s}\right) \in R$, then

3. deleting all $\left(i_{s}, j_{q}\right)$ and $\left(j_{q}, i_{s}\right)$ from $R$,

where $1 \leq s \leq k, s \neq t$ and $1 \leq q \leq h$.

Let $R_{S}^{r 1}$ denote the new relation and $A_{S}^{r 1}=\left\{i_{t}\right\} \cup(A \backslash S)$, then $\left(A_{S}^{r 1}, R_{S}^{r 1}\right)$ is a new argumentation framework called the reduced argumentation framework from $A F$ wrt $r 1$ and $S$. Obviously, the reduced matrix $M_{S}^{r 1}(A F)$ is exactly the matrix of $\left(A_{S}^{r 1}, R_{S}^{r 1}\right)$.

Theorem 7 Given $A F=(A, R)$ with $A=\{1,2, \ldots, n\}$. Let $S=\left\{i_{1}, i_{2}, \ldots, i_{k}\right\} \subseteq A$ be conflict-free and $1 \leq t \leq k$. Then, $S$ is stable (respectively admissible, complete, preferred) in $A F$ if and only if $\left\{i_{t}\right\}$ is stable (respectively admissible, complete, preferred) in the reduced argumentation framework $\left(A_{S}^{r 1}, R_{S}^{r 1}\right)$.

Example 5 Let $A F=(A, R)$ be an argumentation framework with $A=\{1,2,3,4\}$ and $R=\{(1,3),(2,4),(3,1),(3,2),(4,2)\}$. The graph and matrix of AF are as follows:

$$
M(A F)=\left(\begin{array}{cccc}
0 & 0 & 1 & 0 \\
0 & 0 & 0 & 1 \\
1 & 1 & 0 & 0 \\
0 & 1 & 0 & 0
\end{array}\right)
$$

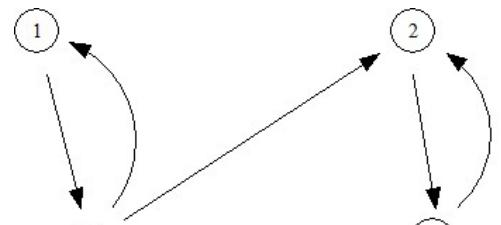

(3)

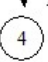

Since $S=\{1,2\}$ is conflict-free, we can turn $M(A F)$ into the following reduced matrix according to the above rules ( $S$ is compacted into $\{2\}$ ):

$$
M_{S}^{r 1}(A F)=\left(\begin{array}{lll}
0 & 1 & 1 \\
1 & 0 & 0 \\
1 & 0 & 0
\end{array}\right) .
$$

The corresponding reduced argumentation framework is $\left(A_{S}^{r 1}, R_{S}^{r 1}\right)$ where $A_{S}^{r 1}=\{2,3,4\}$ and $R_{S}^{r 1}=\{(2,3),(2,4),(3,2),(4,2)\}$. The graph of $\left(A_{S}^{r 1}, R_{S}^{r 1}\right)$ is the following one. 


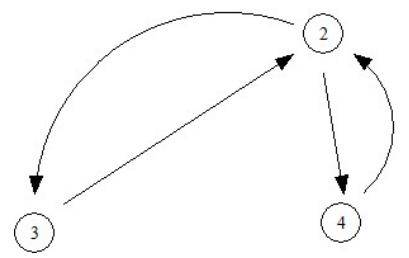

Note that $S=\{1,2\}$ is stable in $A F$ and $\{2\}$ is stable in $\left(A_{S}^{r 1}, R_{S}^{r 1}\right)$.

\subsection{The second type of reduction}

By Theorem 7, we can contract a conflict-free subset $S$ into a single argument when we try to determine whether it is an extension. Generally speaking, there may be many conflictfree subsets in an argumentation framework. In this part, we will develop the above process for two or more conflict-free subsets and turn the matrix of an argumentation framework into a reduced matrix of less order, from which the determination of various extensions becomes more efficient. Of cause, we only need to discuss the case of two conflict-free subsets. First, let us discuss the reduction wrt two disjoint conflict-free subsets.

Let $A F=(A, R)$ be an argumentation framework with $A=\{1,2, \ldots, n\}$ and $M(A F)$ be the matrix of $A F$. Let $S_{1}=\left\{i_{1}, i_{2}, \ldots, i_{k}\right\}$ and $S_{2}=\left\{j_{1}, j_{2}, \ldots, j_{h}\right\}$ be two conflict-free subsets of $A$ such that $S_{1} \cap S_{2}=\emptyset$, our purpose is to contract $S_{1}, S_{2}$ into two different entries in the matrix so that the sub-blocks and the related entries have the same status wrt semantics.

We reduce the matrix $M(A F)$ into another matrix $M_{S_{1}, S_{2}}^{r 2}(A F)$ of order $n-k-h+2$ by the following rules:

Let $1 \leq t \leq k$ and $1 \leq s \leq h$,

1. for $S_{1}$, adding every row $i_{p}$ to the row $i_{t}$, adding every column $i_{p}$ to the column $i_{t}$,

2. for $S_{2}$, doing the similar work as for $S_{1}$, then

3. deleting every row $i_{p}$ and column $i_{p}$,

4. deleting every row $j_{q}$ and column $j_{q}$,

where $1 \leq p \leq k, 1 \leq q \leq h$ and $p \neq t, q \neq s$.

The matrix $M_{S_{1}, S_{2}}^{r 2}(A F)$ is called the reduced matrix wrt $r 2$ and the conflict-free subsets $S_{1}$ and $S_{2}$, or the reduced matrix wrt $S_{1}$ and $S_{2}$ for short.

Corresponding to the above process for matrix, we can reduce the original argumentation framework $A F$ into a new one with $n-k-h+2$ arguments. Let $1 \leq t \leq k$ and $1 \leq s \leq h$, we apply the following rules:

1. adding $\left(i_{t}, i\right)$ to $R$ if $\left(i_{p}, i\right) \in R$, adding $\left(i, i_{t}\right)$ to $R$ if $\left(i, i_{p}\right) \in R$, 
2. adding $\left(j_{s}, j\right)$ to $R$ if $\left(j_{q}, j\right) \in R$, adding $\left(j, j_{s}\right)$ to $R$ if $\left(j, j_{q}\right) \in R$,

3. deleting all $\left(i_{p}, i\right)$ and $\left(i, i_{p}\right)$ from $R$,

4. deleting all $\left(j_{q}, j\right)$ and $\left(j, j_{q}\right)$ from $R$,

where $i \in A \backslash S_{1}, j \in A \backslash S_{2}, 1 \leq p \leq k, 1 \leq q \leq h$ and $p \neq t, q \neq s$.

Let $R_{S_{1}, S_{2}}^{r 2}$ denote the new relation and $A_{S_{1}, S_{2}}^{r 2}=\left\{i_{t}, j_{s}\right\} \cup\left(A \backslash\left(S_{1} \cup S_{2}\right)\right)$, then $\left(A_{S_{1}, S_{2}}^{r 2}, R_{S_{1}, S_{2}}^{r 2}\right)$ is a new argumentation framework called the reduced argumentation framework from $A F$ wrt $r 2$ and $\left(S_{1}, S_{2}\right)$. Obviously, $M_{S_{1}, S_{2}}^{r 2}(A F)$ is exactly the matrix of $\left(A_{S_{1}, S_{2}}^{r 2}, R_{S_{1}, S_{2}}^{r 2}\right)$.

Theorem 8 Given $A F=(A, R)$ with $A=\{1,2, \ldots, n\}$. Let $S_{1}=\left\{i_{1}, i_{2}, \ldots, i_{k}\right\}$ and $S_{2}=$ $\left\{j_{1}, j_{2}, \ldots, j_{h}\right\}$ be two conflict-free subsets of $A F$ such that $S_{1} \cap S_{2}=\emptyset$. Let $1 \leq t \leq k$ and $1 \leq s \leq h$, then

- $S_{1}$ is stable (respectively admissible, complete, preferred) in AF if and only if $\left\{i_{t}\right\}$ is stable (respectively admissible, complete, preferred) in $\left(A_{S_{1}, S_{2}}^{r 2}, R_{S_{1}, S_{2}}^{r 2}\right)$,

- $S_{2}$ is stable (respectively admissible, complete, preferred) in $A F$ if and only if $\left\{j_{s}\right\}$ is stable (respectively admissible, complete, preferred) in $\left(A_{S_{1}, S_{2}}^{r 2}, R_{S_{1}, S_{2}}^{r 2}\right)$.

Example 5 (cont'd):

$$
M(A F)=\left(\begin{array}{cccc}
0 & 0 & 1 & 0 \\
0 & 0 & 0 & 1 \\
1 & 1 & 0 & 0 \\
0 & 1 & 0 & 0
\end{array}\right)
$$

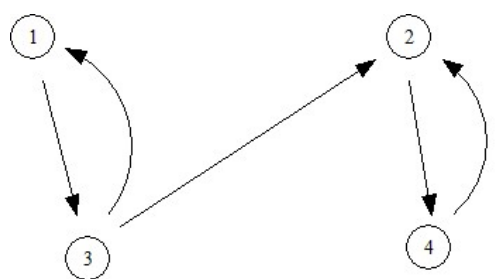

Since $\{1,2\}$ and $\{3,4\}$ are disjoint conflict-free sets, we can turn $M(A F)$ into the following reduced matrix according to the above rules:

$$
M_{S_{1}, S_{2}}^{r 2}(A F)=\left(\begin{array}{cc}
0 & 1 \\
1 & 0
\end{array}\right)
$$

Here, $\left(A_{S_{1}, S_{2}}^{r 2}, R_{S_{1}, S_{2}}^{r 2}\right)$ is the reduced argumentation framework with $A_{S_{1}, S_{2}}^{r 2}=\{1,3\}$ and $R_{S_{1}, S_{2}}^{r 2}=\{(1,3),(3,1)\}$. The graph of $\left(A_{S_{1}, S_{2}}^{r 2}, R_{S_{1}, S_{2}}^{r 2}\right)$ is as follows.

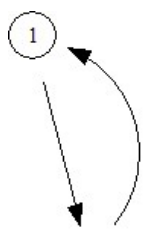

(3)

Note that $\{1\}$ and $\{3\}$ are stable in the reduced argumentation framework $\left(A_{S_{1}, S_{2}}^{r 2}, R_{S_{1}, S_{2}}^{r 2}\right)$, meanwhile $\{1,2\}$ and $\{3,4\}$ are stable in $A F$. 


\subsection{The third type of reduction}

For two conflict-free subsets with non-empty intersection, it seems a little complicated to contract the matrix of $A F$. We can contract each conflict-free subset into a single argument. But, these two arguments must not be the same. Otherwise, we will contract the union of these two conflict-free subsets into a single argument, which may not be conflict-free. Therefore, it is not suitable for our purpose to contract these two conflict-free subsets into the same argument.

Let $A F=(A, R)$ be an argumentation framework with $A=\{1,2, \ldots, n\}$ and $M(A F)$ be the matrix of $A F$. Let $S_{1}=\left\{i_{1}, i_{2}, \ldots, i_{k}\right\}$ and $S_{2}=\left\{j_{1}, j_{2}, \ldots, j_{h}\right\}$ be two conflict-free subsets of $A F$ such that $S_{1} \backslash S_{2} \neq \emptyset$ and $S_{1} \backslash S_{2} \neq \emptyset$. Once again, we try to contract $S_{1}, S_{2}$ into two different entries in the matrix, and ensure that the sub-blocks and the related entries have the same status wrt semantics.

We reduce the matrix $M(A F)$ into another matrix $M_{S_{1}, S_{2}}^{r 3}(A F)$ of less order by the following rules:

Let $1 \leq t \leq k$ and $1 \leq s \leq h$ such that $i_{t} \neq j_{s}$,

1. for $S_{1}$, adding every row $i_{p}$ to the row $i_{t}$, adding every column $i_{p}$ to the column $i_{t}$,

2. for $S_{2}$, doing the similar work as for $S_{1}$, then

3. deleting every row $i_{p}$ and column $i_{p}$,

4. deleting every row $j_{q}$ and column $j_{q}$,

where $1 \leq p \leq k, 1 \leq q \leq h$ and $p \neq t, q \neq s$.

The matrix $M_{S_{1}, S_{2}}^{r 3}(A F)$ is called the reduced matrix wrt $r 3$ and the conflict-free subsets $S_{1}$ and $S_{2}$, or the reduced matrix wrt $S_{1}$ and $S_{2}$ for short.

Corresponding to the above process for matrix, we can reduce the argumentation framework $A F$ into a new one with less arguments. Let $1 \leq t \leq k$ and $1 \leq s \leq h$ such that $i_{t} \neq j_{s}$, we apply the following rules to the $A F$ :

1. adding $\left(i_{t}, i\right)$ to $R$ if $\left(i_{p}, i\right) \in R$, adding $\left(i, i_{t}\right)$ to $R$ if $\left(i, i_{p}\right) \in R$,

2. adding $\left(j_{s}, j\right)$ to $R$ if $\left(j_{q}, j\right) \in R$, adding $\left(j, j_{s}\right)$ to $R$ if $\left(j, j_{q}\right) \in R$,

3. deleting all $\left(i_{p}, i\right)$ and $\left(i, i_{p}\right)$ from $R$,

4. deleting all $\left(j_{q}, j\right)$ and $\left(j, j_{q}\right)$ from $R$,

where $i \in A \backslash S_{1}, j \in A \backslash S_{2}, 1 \leq p \leq k, 1 \leq q \leq h$ and $p \neq t, q \neq s$.

Let $R_{S_{1}, S_{2}}^{r 3}$ denote the new relation and $A_{S_{1}, S_{2}}^{r 3}=\left\{i_{t}, j_{s}\right\} \cup\left(A \backslash\left(S_{1} \cup S_{2}\right)\right)$, then $\left(A_{S_{1}, S_{2}}^{r 3}, R_{S_{1}, S_{2}}^{r 3}\right)$ is a new argumentation framework called the reduced argumentation framework from $A F$ wrt $r 3$ and $\left(S_{1}, S_{2}\right)$. Obviously, $M_{S_{1}, S_{2}}^{r 3}(A F)$ is exactly the matrix of $\left(A_{S_{1}, S_{2}}^{r 3}, R_{S_{1}, S_{2}}^{r 3}\right)$. 
Theorem 9 Given $A F=(A, R)$ with $A=\{1,2, \ldots, n\}$. Let $S_{1}=\left\{i_{1}, i_{2}, \ldots, i_{k}\right\}$ and $S_{2}=$ $\left\{j_{1}, j_{2}, \ldots, j_{h}\right\}$ be two conflict-free subsets of $A$ such that $S_{1} \backslash S_{2} \neq \emptyset$ and $S_{1} \backslash S_{2} \neq \emptyset$. Let $1 \leq t \leq k$ and $1 \leq s \leq h$ such that $i_{t} \neq j_{s}$. Then,

- $S_{1}$ is stable (respectively admissible, complete, preferred) in AF if and only if $\left\{i_{t}\right\}$ is stable (respectively admissible, complete, preferred) in $\left(A_{S_{1}, S_{2}}^{r 3}, R_{S_{1}, S_{2}}^{r 3}\right)$

- $S_{2}$ is stable (respectively admissible, complete, preferred) in AF if and only if $\left\{j_{s}\right\}$ is stable (respectively admissible, complete, preferred) in $\left(A_{S_{1}, S_{2}}^{r 3}, R_{S_{1}, S_{2}}^{r 3}\right)$

Example 5 (cont'd): $M(A F)=\left(\begin{array}{llll}0 & 0 & 1 & 0 \\ 0 & 0 & 0 & 1 \\ 1 & 1 & 0 & 0 \\ 0 & 1 & 0 & 0\end{array}\right)$

Since $\{1,2\}$ and $\{1,4\}$ are conflict-free sets, we can turn $M(A F)$ into the following reduced matrix according to the above rules:

$$
M_{S_{1}, S_{2}}^{r 3}(A F)=\left(\begin{array}{ccc}
0 & 1 & 1 \\
1 & 0 & 1 \\
1 & 1 & 0
\end{array}\right)
$$

Here, $\left(A_{S_{1}, S_{2}}^{r 3}, R_{S_{1}, S_{2}}^{r 3}\right)$ is the reduction argumentation framework with $A_{S_{1}, S_{2}}^{r 3}=\{2,3,4\}$ and $R_{S_{1}, S_{2}}^{r 3}=\{(2,3),(2,4),(3,2),(3,4),(4,2),(4,3)\}$.

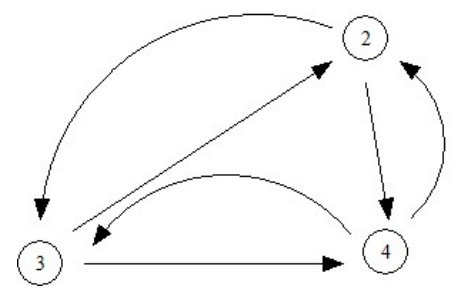

Note that, $\{2\}$ is stable in $\left(A_{S_{1}, S_{2}}^{r 3}, R_{S_{1}, S_{2}}^{r 3}\right)$ meanwhile $\{1,2\}$ is stable in $A F$, and $\{4\}$ is stable in $\left(A_{S_{1}, S_{2}}^{r 3}, R_{S_{1}, S_{2}}^{r 3}\right)$ as $\{1,4\}$ is also stable in $A F$.

By now, we can determine the stable, admissible and complete extensions by the matrix approach of argumentation frameworks. And, it is more efficient if we combine the standard form with the reduction form of matrix. But, what we should have to face is that whether we can also determine the grounded and preferred extensions by the matrix approach. Note that, maximality is the most essential feature of preferred semantics. From this point, we propose another kind of reduction of matrix for argumentation frameworks, which is based on the division of argumentation frameworks. 


\section{Matrix reduction based on the division of $A F$}

The division of argumentation frameworks into sub-argumentation frameworks has already been considered [19] for handling dynamics of argumentation frameworks. Indeed many problems in argumentation frameworks can be dealt with by the division of $A F$. For example, the grounded extension can be viewed as the union of two subsets $I_{A F}$ and $E$ : $I_{A F}$ consists of the initial arguments of $A F$ and $E$ is the grounded extension of the remaining sub-argumentation $\left(B,\left.R\right|_{B}\right)$ where $B=A \backslash\left(I_{A F} \cup R^{+}\left(I_{A F}\right)\right)$. A preferred extension coincides with an admissible extension $E$ from which the associated remaining sub-argumentation $\left(C,\left.R\right|_{C}\right)$ has no nonempty admissible extension according to the maximality of preferred extension, where $C=A \backslash\left(E \cup R^{+}(E)\right)$.

Since a sub-argumentation framework has relationship with other parts of a given argumentation framework, there is no sense to study a sub-argumentation framework independently. But, if we have had some special subset such as an unattacked subset, or we only need to treat some special extensions, for example, a preferred extension containing a known admissible subset, then the study of the remaining sub-argumentation framework would be of great importance. Under this circumstance, the matrix of the subargumentation framework is another kind of reduced matrix for the original argumentation framework, compared with the one in the previous section. Next, we will mainly talk about this kind of reduction for two purposes: given a nonempty admissible extension, find other admissible, preferred, complete extensions containing it; given an unattacked subset, find all the grounded, admissible, preferred, complete extensions.

\subsection{Building the grounded and preferred extensions}

Recall that the grounded and preferred extensions have the feature of minimality and maximality respectively. So, it is difficult to determine them directly without any comparison process. Thanks to another definition of grounded extension by characteristic functions, we can turn our attention to another side of it. In fact, the grounded extension can be obtained by starting from the subset of initial arguments, then continuing this process with its remaining sub-argumentation, and so on. Similarly, preferred extensions can be obtained by replacing the subset of initial arguments by an admissible set.

Corresponding to every extension $S$ of an argumentation framework $A F=(A, R)$, there is a sub-block $M^{c f}(S)$ in the matrix $M(A F)$. For the remaining sub-argumentation $A F_{1}=\left(B,\left.R\right|_{B}\right)$ with $B=A \backslash\left(S \cup R^{+}(S)\right)$, there is also a correspondence between the extension $T$ of $A F_{1}$ and the sub-block $M^{c f}(T)$ of $M\left(A F_{1}\right)$. In order to determine the extensions of $A F_{1}$, we can apply the theorems obtained in Section 4. So, the matrix $M\left(A F_{1}\right)$ becomes an object in the central position of study. We call it the reduced matrix wrt extension $S$.

For each extension $T$ of $A F_{1}$, we can turn the matrix $M(A F)$ into a standard form wrt $S \cup T$ by a sequence of dual interchanges. Based on the results obtained in Section 4, we 
can deduce the following theorem.

Theorem 10 Let $A F=(A, R)$ be an argumentation framework, $S \subseteq A$ be an admissible extension of $A F$, and $B=A \backslash\left(S \cup R^{+}(S)\right)$. If $T \subseteq B$ is an admissible extension of the remaining sub-argumentation $\left.A F\right|_{B}$ wrt $S$, then $S \cup T$ is an admissible extension of $A F$.

Remark 7 : If we let $T$ to be a stable (resp. complete, preferred) extension in Theorem 10, then $S \cup T$ will be a stable (resp. complete, preferred) extension of $A F$.

Example 6 Let $A F=(A, R)$ be an argumentation framework with $A=\{1,2,3,4\}$ and $R=\{(1,4),(2,3),(3,4),(4,2),(4,3)\}$. The graph and matrix of $A F$ are as follows.

$$
M(A F)=\left(\begin{array}{cccc}
0 & 0 & 0 & 1 \\
0 & 0 & 1 & 0 \\
0 & 0 & 0 & 1 \\
0 & 1 & 1 & 0
\end{array}\right)
$$

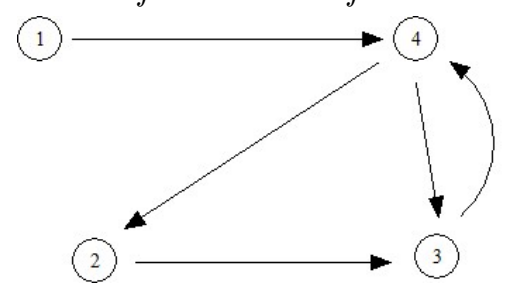

Obviously, $S=\{1\}$ is an admissible extension of $A F, R^{+}(S)=\{4\}$ and $B=A \backslash(S \cup$ $\left.R^{+}(S)\right)=\{2,3\}$. So, the graph and matrix of the remaining sub-argumentation $\left.A F\right|_{B}$ wrt $S$ are as follows.

$$
M\left(\left.A F\right|_{B}\right)=\left(\begin{array}{ll}
0 & 1 \\
0 & 0
\end{array}\right)
$$

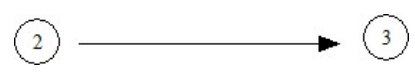

It is easy to see that $T=\{2\}$ is admissible in the remaining sub-argumentation $\left.A F\right|_{B}$ wrt $S$. By Theorem 10, we conclude that $S \cup T=\{1,2\}$ is admissible in AF.

Building the grounded extension It is well known that the grounded extension can be built incrementally starting from the initial arguments. Then the arguments attacked by them can be removed, resulting in a modified argumentation framework. In turn the arguments attacked by the new initial arguments can be removed, and so on. The process stops when no new initial argument arises after a removal step. The set of all initial arguments identified so far is the grounded extension.

The above description indicates a feature of the grounded semantics: Comparing with the semantics we discussed above, we may consider grounded semantics as a kind of semantics based on recurrence.

If $A F$ has no initial argument, then the grounded extension $S$ of $A F$ is empty. Otherwise, let $I_{1}$ be the set of initial arguments of $A F$, then $I_{1}$ is an admissible extension of $A F$. Next, we consider the sub-argumentation framework $\left.A F\right|_{B_{1}}$ where $B_{1}=A \backslash\left(I_{1} \cup R^{+}\left(I_{1}\right)\right)$. If it has no initial argument, then the grounded extension $S=I_{1}$. Otherwise, let $I_{2}$ be the set of initial arguments of $\left.A F\right|_{B_{1}}$ and $B_{2}=B_{1} \backslash\left(I_{2} \cup R^{+}\left(I_{2}\right)\right)$. By Theorem 10, $I_{1} \cup I_{2}$ 
is an admissible extension of $A F$. This process can be done repeatedly, until some $\left.A F\right|_{B_{t}}$ has no initial argument, where $1 \leq t \leq n$. It is easy to verify that $S=I_{1} \cup \ldots \cup I_{t}$ is the grounded extension of $A F$.

\section{Example 6 (cont'd):}

$$
M(A F)=\left(\begin{array}{llll}
0 & 0 & 0 & 1 \\
0 & 0 & 1 & 0 \\
0 & 0 & 0 & 1 \\
0 & 1 & 1 & 0
\end{array}\right)
$$

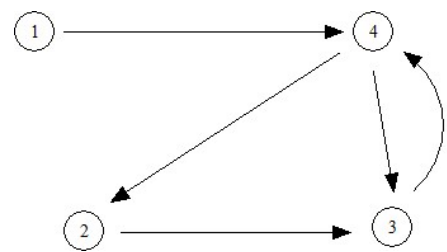

Obviously, $I_{1}=\{1\}$ is an admissible extension of $A F, R^{+}\left(I_{1}\right)=\{4\}$ and $B_{1}=A \backslash\left(I_{1} \cup\right.$ $\left.R^{+}\left(I_{1}\right)\right)=\{2,3\}$. So, the graph and matrix of the remaining sub-argumentation $\left.A F\right|_{B_{1}}$ wrt $I_{1}$ are as follows.

$$
M\left(\left.A F\right|_{B_{1}}\right)=\left(\begin{array}{ll}
0 & 1 \\
0 & 0
\end{array}\right)
$$

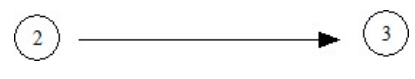

It is easy to see that $I_{2}=\{2\}$ is admissible in $\left.A F\right|_{B_{1}}$, and $I_{1} \cup I_{2}=\{1,2\}$ is admissible in $\left.A F\right|_{B_{1}}$. Since $B_{2}=B_{1} \backslash\left(I_{2} \cup R^{+}\left(I_{2}\right)\right)=\emptyset$, we conclude that $S=I_{1} \cup I_{2}=\{1,2\}$ is grounded in $A F$

Building the preferred extensions A preferred extension is defined as a maximal element (wrt set inclusion) of the set of admissible extensions. So, it can be also built incrementally starting from some admissible extension. So the preferred semantics may also be viewed as a semantics based on recurrence.

Let $S_{1}$ be any admissible extension of $A F$, and $B_{1}=A \backslash\left(S_{1} \cup R^{+}\left(S_{1}\right)\right)$. If $B_{1}=\emptyset$ or the sub-argumentation framework $\left.A F\right|_{B_{1}}$ does not have nonempty admissible extension, then $S_{1}$ is a preferred extension of $A F$. Otherwise, let $S_{2}$ be an nonempty admissible extension. Then, $S_{1} \cup S_{2}$ is an admissible extension of $A F$ by Theorem 10. Let $B_{2}=B_{1} \backslash\left(S_{2} \cup R^{+}\left(S_{2}\right)\right)$, then it is a sub-argumentation framework of $\left.A F\right|_{B_{1}}$. This process can be done repeatedly, until some sub-argumentation framework $\left.A F\right|_{B_{s}}$ has no nonempty admissible extension where $1 \leq s \leq n$. It is easy to verify that $S_{1} \cup \ldots \cup S_{t}$ is a preferred extension of $A F$.

Note that, the preferred extension we obtained in the above process depends not only on the choice of the initial admissible extension but also on the selection of the nonempty admissible in each sub-argumentation framework. Namely, starting from different initial admissible extensions we may get different preferred extensions. Even for a fixed initial admissible extension, selecting different nonempty admissible extensions in each sub-argumentation framework may also result in different preferred extensions.

Example 7 Let $A F=(A, R)$ be an argumentation framework with $A=\{1,2,3,4,5\}$ and $R=\{(1,5),(2,3),(2,5),(3,4),(4,2),(4,5),(5,2)\}$. The graph and matrix of AF are as follows. 


$$
M(A F)=\left(\begin{array}{ccccc}
0 & 0 & 0 & 0 & 1 \\
0 & 0 & 1 & 0 & 1 \\
0 & 0 & 0 & 1 & 0 \\
0 & 1 & 0 & 0 & 1 \\
0 & 1 & 0 & 0 & 0
\end{array}\right)
$$

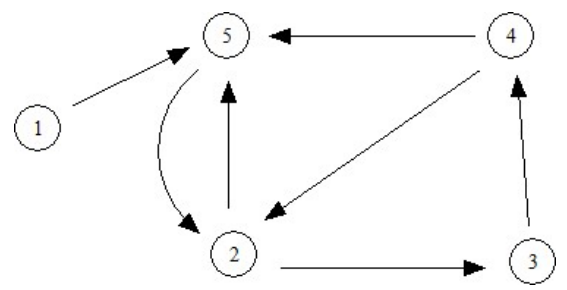

Obviously, $S=\{1\}$ is an admissible extension of $A F, R^{+}(S)=\{5\}$ and $B=A \backslash(S \cup$ $\left.R^{+}(S)\right)=\{2,3,4\}$. So, the graph and matrix of the remaining sub-argumentation $\left.A F\right|_{B}$ wrt $S$ are as follows.

$$
M\left(\left.A F\right|_{B}\right)=\left(\begin{array}{ccc}
0 & 1 & 0 \\
0 & 0 & 1 \\
1 & 0 & 0
\end{array}\right)
$$

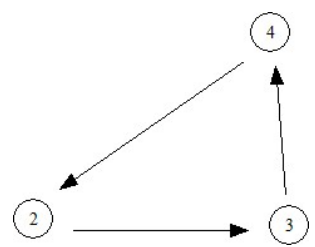

It is easy to see that there is no nonempty admissible in the remaining sub-argumentation $\left.A F\right|_{B}$. So we conclude that $S=\{1\}$ is preferred in $A F$.

Remark 8 If we want to determine whether a conflict-free subset $S$ is a preferred extension of $A F$, the maximality of $S$ (wrt set inclusion) must be verified. One may divide AF into two parts: $S$ and $B=A \backslash\left(S \cup R^{+}(S)\right)$ and study the remaining sub-argumentation $\left(B,\left.R\right|_{B}\right)$. If the remaining sub-argumentation has no nonempty admissible extension, then $S$ is preferred. But, the drawback is that the remaining sub-argumentation may be also very large and we have no efficient method to determine the existence of nonempty admissible extensions by now.

\subsection{Handling the dynamics argumentation framework}

In recent years, the research on dynamics argumentation framework has become more and more active $[11,12,5,6,10,17,19,24]$. In [12], Cayrol et al. introduced change operations to describe the dynamics of argumentation frameworks, and systematically studied the structural properties for change operations. Based on these notions, Liao et al.[19] concentrated their attention on the directionality of argumentation frameworks and constructed a division-based method for dynamics of argumentation frameworks. In the following, we introduce the reduction of a matrix wrt an extension in an unattacked subset of the argumentation framework and give the decomposition theorem of extensions.

Directionality is a basic principle for extension-based semantics. According to [1, 2], the following semantics have been proved to satisfy the directionality criterion: grounded semantics, complete semantics, preferred semantics, ideal semantics, and the prudent version of grounded semantics. Directionality is based on the unattacked subsets. So, we recall the definition of unattacked subset. 
Definition 11 [2] Given an argumentation framework $A F=(A, R)$, a non-empty set $S \in A$ is unattacked if and only if there is no $a \in A \backslash S$ such that a attacks $S$.

Given an unattacked subset $U$ of an argumentation framework $A F$. Let $E_{1}$ be an admissible extension in the sub-argumentation $\left.A F\right|_{U}$, then we have the remaining subargumentation $\left.A F\right|_{T}$ with $T=A \backslash\left(E_{1} \cup R^{+}\left(E_{1}\right)\right)$. If $E_{2}$ is an admissible extension of $\left.A F\right|_{T}$, then $M^{c f}\left(E_{2}\right)$ is the corresponding sub-block in $M\left(\left.A F\right|_{T}\right)$. In order to determine the extensions of $\left.A F\right|_{T}$, we can apply the theorems obtained in Section 4. So, the matrix $M\left(\left.A F\right|_{T}\right)$ becomes the main object we need to face. We call it the reduced matrix wrt extension $E_{1}$.

For each admissible extension $E_{2}$, we can turn the matrix $M(A F)$ into a standard form wrt $E_{1} \cup E_{2}$ by a sequence of dual interchanges. Based on the results obtained in Section 4 , we can derive the following theorem.

Theorem 11 Let $A F=(A, R)$ be an argumentation framework and $U$ an unattacked subset of $A$, then $E \subseteq A$ is an admissible subset of $A F$ if and only if $E_{1}=E \cap U$ is an admissible extension of the sub-argumentation $\left.A F\right|_{U}$ and $E_{2}=E \cap T$ is an admissible extension of the remaining sub-argumentation $\left.A F\right|_{T}$ wrt $E_{1}$, where $T=A \backslash\left(E_{1} \cup R^{+}\left(E_{1}\right)\right)$.

Remark 9 From the proof, it is not difficult to see that Theorem 11 is also true for other extensions which satisfy the directionality principle. Namely, we can replace "admissible" by "complete, preferred, grounded or ideal".

Remark 10 For any unattacked subset $U$ of AF, there may be many admissible extensions in the sub-argumentation framework $\left.A F\right|_{U}$. Each admissible extension $E_{1}$ will produce a remaining sub-argumentation framework $\left.A F\right|_{T}$ with $T=A \backslash\left(E_{1} \cup R^{+}\left(E_{1}\right)\right)$, and thus combining $E_{1}$ with every admissible extension of $\left.A F\right|_{T}$ can give us an admissible extension in AF. We may say that the family of admissible extensions in $\left.A F\right|_{T}$ is determined by $E_{1}$.

Example 8 Let $A F=(A, R)$ be an argumentation framework with $A=\{1,2,3,4,5\}$ and $R=\{(1,2),(2,1),(2,3),(2,5),(4,3),(4,5),(5,4)\}$. The matrix and graph are as follows.

$$
M(A F)=\left(\begin{array}{ccccc}
0 & 1 & 0 & 0 & 0 \\
1 & 0 & 1 & 0 & 1 \\
0 & 0 & 0 & 0 & 0 \\
0 & 0 & 1 & 0 & 1 \\
0 & 0 & 0 & 1 & 0
\end{array}\right)
$$

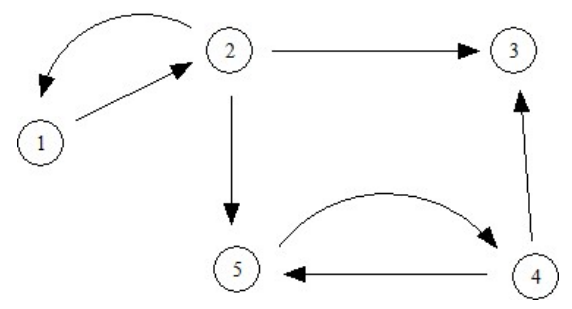


It is easy to check that $U=\{1,2\}$ is an unattacked subset of $A F$, and $E_{1}=\{1\}$ is an admissible extension in the sub-argumentation $\left.A F\right|_{U}$. Since $T=A \backslash\left(E_{1} \cup R^{+}\left(E_{1}\right)\right)=$ $\{3,4,5\}$, the matrix and graph of the remaining sub-argumentation $\left.A F\right|_{T}$ wrt $E_{1}$ are as follows:

$$
M\left(\left.A F\right|_{T}=\left(\begin{array}{ccc}
0 & 0 & 0 \\
1 & 0 & 1 \\
0 & 1 & 0
\end{array}\right)\right.
$$

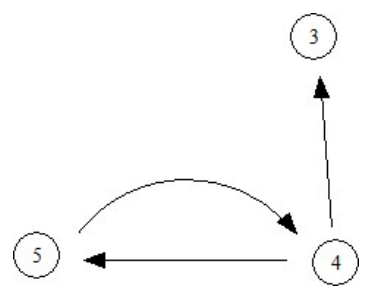

By Theorem 3, we can identify that $\{4\},\{5\}$ and $\{3,5\}$ are all the admissible extensions in $\left.A F\right|_{T}$. According to Theorem 11, $\{1,4\},\{1,5\}$ and $\{1,3,5\}$ are all the admissible extensions in $A F$ determined by $E_{1}=\{1\}$ in $\left.A F\right|_{U}$.

Obviously, Theorem 11 is a general result for argumentation frameworks. But it happens that this result plays a very important role when applying it to dynamics of argumentation frameworks. In order to describe this application, we need to present basic notions related to dynamics of argumentation frameworks. We focus on the work described in [19].

Let $U_{\text {arg }}$ be the universe of arguments, $A F=(A, R)$ be an argumentation framework. Different kinds of change on $A F$ can be considered: (1) adding (or deleting) a set of interactions between the arguments in $A$, we denote this set by $\mathcal{I}_{A}$. (2) adding a set $B \subseteq U_{\text {arg }} \backslash A$ of arguments, we can also add some interactions related to it, including a set of interactions between $A$ and $B$ and a set of interactions between the arguments in $B$. The union of these two sets of interactions is denoted by $\mathcal{I}_{A: B}$. (3) deleting a set $B \subseteq A$ of arguments, we will also delete all the interactions related to it, including the set of interactions between $A \backslash B$ and $B$ and the set of interactions between the arguments in $B$. The union of these two sets of interactions is denoted by $\mathcal{I}_{A \backslash B: B}$. (4) after deleting the set $B \subseteq A$ of arguments, we can continue to delete some interactions between the arguments in $A \backslash B$. This set of interactions is denoted by $\mathcal{I}_{A \backslash B}$, similar as in (1).

According to $[12,19]$, an addition on $A F$ is represented as a tuple $\left(B, I_{A: B} \cup I_{A}\right)$ with $B \subseteq U_{\text {arg }} \backslash A$, and a deletion on $A F$ is represented as a tuple $\left(B, I_{A \backslash B: B} \cup I_{A \backslash B}\right)$ with $B \subseteq A$.

Definition 12 [19] Let $A F=(A, R)$ be an argumentation framework, $\left(B, I_{A: B} \cup I_{A}\right)$ be an addition and $\left(B, I_{A \backslash B: B} \cup I_{A \backslash B}\right)$ be a deletion. The updated $A F$ wrt $\left(B, I_{A: B} \cup I_{A}\right)$ and $\left(B, I_{A \backslash B: B} \cup I_{A \backslash B}\right)$ is respectively defined as follows:

$$
\begin{aligned}
& A F^{\oplus}=\left(A^{\oplus}, R^{\oplus}\right)=(A, R) \oplus\left(B, I_{A: B} \cup I_{A}\right)=\left(A \cup B, R \cup I_{A: B} \cup I_{A}\right) \text { and } \\
& A F^{\ominus}=\left(A^{\ominus}, R^{\ominus}\right)=(A, R) \ominus\left(B, I_{A \backslash B: B} \cup I_{A \backslash B}\right)=\left(A \backslash B, R \backslash\left(I_{A \backslash B: B} \cup I_{A \backslash B}\right)\right) .
\end{aligned}
$$

Now, we can apply Theorem 11 to the study of dynamics of argumentation framework. The following two corollaries can be obtained directly. 
Corollary 5 Given an argumentation framework $A F=(A, R)$. Let $A F^{\oplus}$ be the update argumentation framework by adding $\left(B, I_{A: B} \cup I_{A}\right)$, and $U$ be an unattacked subset in $A F^{\oplus}$. If $E_{1}$ is an admissible extension of the sub-argumentation $\left.A F^{\oplus}\right|_{U}$, and $E_{2}$ is an admissible extension of the remaining sub-argumentation $\left.A F^{\oplus}\right|_{T}$ wrt $E_{1}$, where $T=$ $(A \cup B) \backslash\left(E_{1} \cup R^{+}\left(E_{1}\right)\right)$, then $E_{1} \cup E_{2}$ is an admissible extension of $A F^{\oplus}$. Conversely, for each admissible extension $E$ of $A F^{\oplus}, E_{1}=E \cap U$ is an admissible extension of $\left.A F^{\oplus}\right|_{U}$ and $E_{2}=E \cap T$ is an admissible extension of $\left.A F^{\oplus}\right|_{T}$.

Example 9 Let $A F=(A, R)$ be the initial argumentation framework with $A=\{1,2,3,4,5,6\}$ and $R=\{(1,2),(1,3),(2,1),(2,3),(3,4),(5,6),(6,5)\}$. Then, the matrix and graph of $A F$ are as follows:

$$
M(A F)=\left(\begin{array}{cccccc}
0 & 1 & 1 & 0 & 0 & 0 \\
1 & 0 & 1 & 0 & 0 & 0 \\
0 & 0 & 0 & 1 & 0 & 0 \\
0 & 0 & 0 & 0 & 0 & 0 \\
0 & 0 & 0 & 0 & 0 & 1 \\
0 & 0 & 0 & 0 & 1 & 0
\end{array}\right)
$$
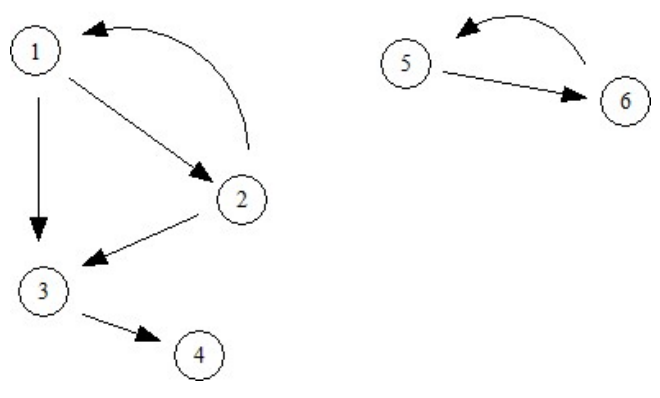

Let $\left(B, I_{A: B} \cup I_{A}\right)$ be an addition with $B=\{7,8\}, I_{A: B}=\{(2,5),(4,7),(7,8),(5,8),(8,6)\}$ and $I_{A}=\{(2,5)\}$. Then, the updated argumentation framework $A F^{\oplus}=(A, R) \oplus\left(B, I_{A: B} \cup\right.$ $\left.\left.I_{A}\right)\right)$ has the following matrix and graph:

$$
M\left(A F^{\oplus}\right)=\left(\begin{array}{llllllll}
0 & 1 & 1 & 0 & 0 & 0 & 0 & 0 \\
1 & 0 & 1 & 0 & 1 & 0 & 0 & 0 \\
0 & 0 & 0 & 1 & 0 & 0 & 0 & 0 \\
0 & 0 & 0 & 0 & 0 & 0 & 1 & 0 \\
0 & 0 & 0 & 0 & 0 & 1 & 0 & 1 \\
0 & 0 & 0 & 0 & 1 & 0 & 0 & 0 \\
0 & 0 & 0 & 0 & 0 & 0 & 0 & 1 \\
0 & 0 & 0 & 0 & 0 & 1 & 0 & 0
\end{array}\right)
$$

Consider the unattacked subset $U=\{1,2,3,4\}$, the matrix and graph of the subargumentation $\left.A F^{\oplus}\right|_{U}$ are as follows: 


$$
M(\{1,2,3,4\})=\left(\begin{array}{cccc}
0 & 1 & 1 & 0 \\
1 & 0 & 1 & 0 \\
0 & 0 & 0 & 1 \\
0 & 0 & 0 & 0
\end{array}\right)
$$

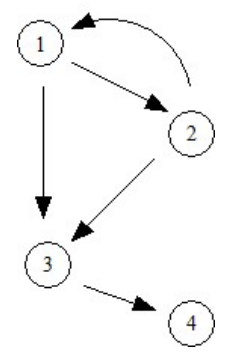

It is easy to check that $E_{1}=\{1,4\}$ is an admissible extension in $\left.A F^{\oplus}\right|_{U}$. Thus, we have $R^{+}\left(E_{1}\right)=\{2,3,7\}$ and $T=\{5,6,8\}$. So, the remaining sub-argumentation $\left.A^{\oplus}\right|_{T}$ wrt $E_{1}$ has the following matrix and graph:

$$
M(\{5,6,8\})=\left(\begin{array}{ccc}
0 & 1 & 1 \\
1 & 0 & 0 \\
0 & 1 & 0
\end{array}\right)
$$

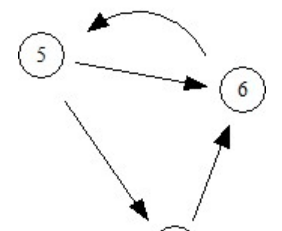

(8)

Obviously, $E_{2}=\{5\}$ is admissible in $\left.A F^{\oplus}\right|_{T}$. And thus, $E_{1} \cup E_{2}=\{1,4,5\}$ is admissible in $A F^{\oplus}$.

Corollary 6 Given an argumentation framework $A F=(A, R)$. Let $A F^{\ominus}$ be the update argumentation framework by deleting $\left(B, I_{A \backslash B: B} \cup I_{A \backslash B}\right)$, and $U$ be an unattacked subset in $A F^{\ominus}$. If $E_{1}$ is an admissible extension of the sub-argumentation $\left.A F^{\ominus}\right|_{U}$, and $E_{2}$ is an admissible extension of the remaining sub-argumentation $\left.A F^{\ominus}\right|_{T}$ wrt $E_{1}$, where $T=A \backslash\left(B \cup E_{1} \cup R^{+}\left(E_{1}\right)\right)$, then $E_{1} \cup E_{2}$ is an admissible extension of $A F^{\ominus}$. Conversely, for each admissible extension $E$ of $A F^{\ominus}, E_{1}=E \cap U$ is an admissible extension of $\left.A F^{\ominus}\right|_{U}$ and $E_{2}=E \cap T$ is an admissible extension of $\left.A F^{\ominus}\right|_{T}$.

Example 10 Let $A F=(A, R)$ be the initial argumentation framework with $A=\{1,2,3,4,5,6\}$ and $R=\{(1,2),(1,3),(2,1),(2,3),(3,4),(4,5),(5,6),(6,5)\}$. Then, the matrix and graph of $A F$ are as follows:

$$
M(A F)=\left(\begin{array}{ccccc}
0 & 1 & 1 & 0 & 0 \\
1 & 0 & 1 & 0 & 0 \\
0 & 0 & 0 & 1 & 0 \\
0 & 0 & 0 & 0 & 1 \\
0 & 0 & 0 & 0 & 0
\end{array}\right)
$$

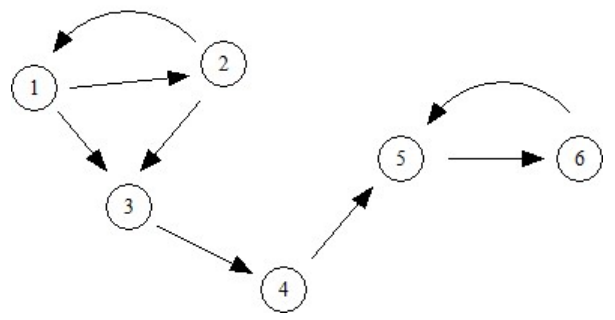

Let $\left(B, I_{A \backslash B: B} \cup I_{A \backslash B}\right)$ be a deletion with $\left.B=\{6\}, I_{A \backslash B: B}=\{(5,6),(6,5))\right\}$ and $I_{A \backslash B}=$ $\{(2,3)\}$. Then, the updated argumentation framework $A F^{\ominus}=(A, R) \ominus\left(B, I_{A \backslash B: B} \cup I_{A \backslash B}\right)$ has the following matrix and graph: 


$$
M\left(A F^{\ominus}\right)=\left(\begin{array}{ccccc}
0 & 1 & 1 & 0 & 0 \\
1 & 0 & 0 & 0 & 0 \\
0 & 0 & 0 & 1 & 0 \\
0 & 0 & 0 & 0 & 1 \\
0 & 0 & 0 & 0 & 0
\end{array}\right)
$$

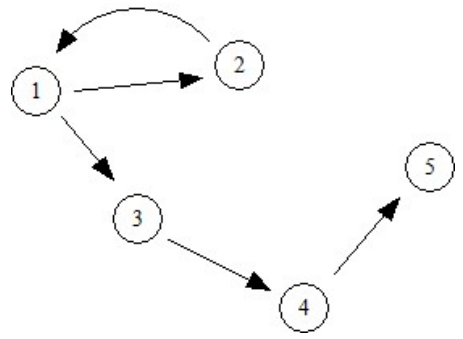

Consider the unattacked subset $U=\{1,2,3\}$, the matrix and graph of the sub-argumentation $\left.A F^{\ominus}\right|_{U}$ are as follows:

$$
M(\{1,2,3\})=\left(\begin{array}{lll}
0 & 1 & 1 \\
1 & 0 & 0 \\
0 & 0 & 0
\end{array}\right)
$$

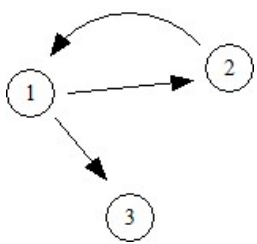

It is easy to check that $E_{1}=\{2\}$ is an admissible extension in $\left.A F^{\ominus}\right|_{U}$. Thus, we have $R^{+}\left(E_{1}\right)=\{1\}$ and $T=\{3,4,5\}$. So, the remaining sub-argumentation $\left.A F^{\ominus}\right|_{T}$ has the following matrix and graph:

$$
M(\{3,4,5\})=\left(\begin{array}{ccc}
0 & 1 & 0 \\
0 & 0 & 1 \\
0 & 0 & 0
\end{array}\right)
$$

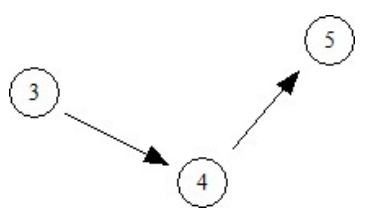

Obviously, $E_{2}=\{3,5\}$ is admissible in $\left.A F^{\ominus}\right|_{T}$. And thus, $E_{1} \cup E_{2}=\{2,3,5\}$ is admissible in $A F^{\ominus}$.

Remark 11 The above two corollaries also hold if we replace "admissible" by "complete, preferred, grounded or ideal". Since they are also based on the division of AF and the directionality principle of AF, they have the similar role as the main results in Liao's paper [19] when applied to dynamics argumentation framework. In fact, these two corollaries are better than Liao's main results[19] when handling the dynamics argumentation framework. First, the remaining sub-argumentation $\left.A F^{\oplus}\right|_{T}$ (or $\left.A F^{\ominus}\right|_{T}$ ) has simpler structure (and so is easier to compute) than the assigned $C A F_{1}\left[E_{1}\right]$ (or $C A F_{2}\left[E_{1}\right]$ ). Furthermore, the condition $C\left(A_{1}\right)\left[E_{1}\right]$ and related interactions increase complexity for computing various extensions in the assigned $C A F_{1}\left[E_{1}\right]$ (or $C A F_{2}\left[E_{1}\right]$ ).

\section{Concluding remarks and future works}

The matrix approach of argumentation frameworks has two important features.

One is in the computational aspect: Given an argumentation framework $A F$, we can assign a matrix $M(A F)$. For any conflict free subset $S$, the matrix $M(A F)$ can be turn into one of the two standard forms by a series of dual interchanges. And thus, determining whether $S$ is an extension can be achieved by checking some sub-blocks related $S$. For 
example, in order to decide whether $S$ is admissible we only need to check whether the subblock $A_{q, k}$ is zero in the standard matrix of $A F$. Corresponding to the standard matrix, the underlying set of an argumentation framework can be divided into three parts. That is the conflict free set $S$, the attacked set $R^{+}(S)$ and the remaining set $A \backslash\left(S \cup R^{+}(S)\right)$. If we want to decide whether $S$ is admissible, the only needed work is to check whether the remaining set $A \backslash\left(S \cup R^{+}(S)\right)$ attacks $S$. In this sense, we may think that the matrix approach is a structural (or integrated) method, which is different from checking the defended status of every argument of $S$.

Another feature is in the theoretical aspect: For any conflict free subset $S$ of an argumentation framework, we can turn the matrix $M(A F)$ into a reduced matrix wrt $S$. This idea is also valid even for two or more conflict-free subsets. And thus, it becomes more efficient to determine extensions using matrices. The reduced matrix wrt conflictfree subsets reflects the idea that an argumentation framework can be compressed into another one with less arguments by contracting conflict-free subsets. This method has not appeared in the literature as we know. For any admissible extension $E$ of an argumentation framework, we can turn the matrix $M(A F)$ into a reduced matrix wrt $E$. Since the reduced matrix wrt an admissible extension relates to the division of an argumentation framework, it can be used to handle topics related to the maximality, directionality and so on. For example, we can compute the preferred extensions, and we can deal with the dynamics of argumentation framework. In fact, we obtain results that are better than the main theorems in [19].

The application of matrix of argumentation frameworks is not limited to the topics of this report. It can also be used to study the structural properties and status-based properties of dynamics argumentation framework defined by Cayrol et al. [12]. This is one direction of our future work. Moreover, as we know, the matrices are successfully used to solve the system of linear equations. We plan to find the equational representation of argumentation frameworks wrt various semantics, by the solution of which we can compute all the extensions we need. A good attempt has been made in this direction by Gabbay [18].

\section{A Appendix: Proofs}

Proof of Theorem 1 Suppose $M^{c f}(S)=0$, then for arbitrary $1 \leq s, t \leq k$ we have that $a_{i_{s}, i_{t}}=0$, i.e., $\left(i_{s}, i_{t}\right) \notin R$. Thus, $S=\left\{i_{1}, i_{2}, \ldots, i_{k}\right\}$ is conflict-free.

Conversely, if $S=\left\{i_{1}, i_{2}, \ldots, i_{k}\right\} \subseteq A$ is conflict-free, then for arbitrary $1 \leq s, t \leq k$ we have that $\left(i_{s}, i_{t}\right) \notin R$, i.e., $a_{i_{s}, i_{t}}=0$. Therefore, we get $M^{c f}(S)=0$.

Proof of Theorem 2 Let $S$ be a stable extension, then every element of $A \backslash S(1 \leq$ $t \leq h)$ is attacked by $S$. Take any column vector $M_{*, j_{t}}(1 \leq t \leq h)$ of the $s$-subblock $M^{s}(S)$, then $j_{t} \in A \backslash S$. By assumption, there is some $i_{r} \in S(1 \leq r \leq k)$ which attacks the 
argument $j_{t}$. It follows that $\left(i_{r}, j_{t}\right) \in R$, and thus we have $a_{i_{r}, j_{t}}=1$ in $M(A F)$. Therefore, the column vector $M_{*, j_{t}}^{s}$ of the $s$-subblock $M^{s}(S)$ is non-zero.

Conversely, suppose each column vector of the $s$-subblock $M^{s}(S)$ is non-zero. Take any element $j_{t} \in A \backslash S(1 \leq t \leq h)$, then $M_{*, j_{t}}^{s}$ is a column vector of the $s$-subblock $M^{s}(S)$. By hypothesis, we know that $M_{*, j_{t}}$ is non-zero. Therefore, there is some $i_{r} \in S(1 \leq r \leq k)$ such that $a_{i_{r}, j_{t}}=1$, and thus $\left(i_{r}, j_{t}\right) \in R$. This means that $j_{t}$ is attacked by the argument $i_{r} \in S$, and thus we prove that $S$ is a stable extension.

Proof of Theorem 3 Assume $S$ is an admissible extension, then every $i_{p} \in S(1 \leq p \leq$ $k$ ) is defended by $S$. If the row vector $M_{t, *}^{a}(1 \leq t \leq h)$ of the $a$-block $M^{a}(S)$ is non-zero, then there is some $i_{r}(1 \leq r \leq k)$ such that $a_{j_{t}, i_{r}}=1$. It follows that $\left(j_{t}, i_{r}\right) \in R$, and thus the argument $i_{r} \in S$ is attacked by the argument $j_{t} \in A \backslash S$. By assumption, $j_{t}$ is attacked by some argument $i_{q} \in S(1 \leq q \leq k)$. This implies that $\left(i_{q}, j_{t}\right) \in R$, and so we have $a_{i_{q}, j_{t}}=1$ in $M(A F)$. Obviously, $a_{i_{q}, j_{t}}$ is an element of the column vector $M_{*, t}^{s}$ of $M^{s}(S)$. Therefore, the column vector $M_{*, t}^{s}$ of the $s$-subblock $M^{s}(S)$ of $S$ is non-zero.

Conversely, suppose any column vector of the $s$-subblock $M^{s}$ of $S$ corresponding to a non-zero row vector of the $a$-subblock $M^{s}(S)$ is non-zero. Let $i_{r} \in S(1 \leq r \leq k)$, and $j_{t} \in A \backslash S(1 \leq t \leq h)$ be any attacker of $i_{r}$. Then, we have $\left(j_{t}, i_{r}\right) \in R$ and thus $a_{j_{t}, i_{r}}=1$ in $M(A F)$. It follows that the row vector $M_{t, *}^{a}$ of the $a$-subblock $M^{a}(S)$ is non-zero. By hypothesis, the corresponding column vector $M_{*, t}^{s}$ of the $s$-subblock $M^{s}(S)$ is non-zero. Therefore, there is some $i_{q}(1 \leq q \leq k)$ such that $a_{i_{q}, j_{t}}=1$ in $M(A F)$. It is immediate that $\left(i_{q}, j_{t}\right) \in R$, and thus the attacker $j_{t}$ of $i_{r}$ is attacked by the argument $i_{q} \in S$. So, $S$ is an admissible extension.

\section{Proof of Theorem 4}

Necessity. Let the column vector $M_{*, t}^{s}$ of the $s$-subblock $M^{s}(S)$ is zero, where $1 \leq t \leq h$. If the corresponding column vector $M_{*, t}^{c}$ of the $c$-subblock $M^{c}(S)$ is zero, then $a_{i, j_{t}}=0$ whenever $1 \leq i \leq n$. This implies that $\left(i, j_{t}\right) \notin R$ for each $1 \leq i \leq n$, and thus the argument $j_{t}$ has no attacker. It follows that $j_{t} \in A \backslash S$ is defended by $S$, a contradiction with the hypothesis. Therefore, the column vector $M_{*, t}^{c} \neq 0$.

Suppose for each nonzero element $a_{j_{q}, j_{t}}(1 \leq q \leq h)$ of $M_{*, t}^{c}$, the corresponding column vector $M_{*, q}^{s}$ of the $s$-subblock $M^{s}(S)$ is non-zero. Let $j_{m}(1 \leq m \leq h)$ be an arbitrary attacker of the argument $j_{t}$, then we have $a_{j_{m}, j_{t}}=1$. It follows that the column vector $M_{*, m}^{s}$ of the $s$-subblock $M^{s}(S)$ is non-zero. Without lost of generality, we may assume that $a_{i_{q}, j_{m}}=1$ for some $1 \leq q \leq k$, then $j_{m}$ is attacked by the argument $i_{q}$. This means that $j_{t}$ is defended by $S$, a contradiction.

Sufficiency. Let $j_{t}(1 \leq t \leq h)$ be any argument of $A \backslash S$, we shall prove that $j_{t}$ is not defended by $S$. If there is some argument $i_{r}(1 \leq r \leq k)$ which attacks $j_{t}$, then we have done. Otherwise, we have $\left(i_{r}, j_{t}\right) \notin R$, i.e., $a_{i_{r}, j_{t}}=0$, for each $1 \leq r \leq k$. This implies that the column vector $M_{*, t}^{s}$ of the $s$-subblock $M^{s}(S)$ is zero. By assumption, the column 
vector $M_{*, t}^{c}$ of $c$-subblock $M^{c}(S)$ is non-zero, and thus there is some element $a_{j_{q}, j_{t}}$ of the column vector $M_{*, t}^{c}$ such that $a_{j_{q}, j_{t}}=1$ and the corresponding column vector $M_{*, q}^{s}$ of the $s$-subblock $M^{s}(S)$ is zero. This indicates that $\left(j_{q}, j_{t}\right) \in R$ and $a_{i_{r}, j_{q}}=0$ for each $1 \leq r \leq k$. Therefore, the attacker $j_{q}$ of $j_{t}$ is not defeated by $S$, and thus $j_{t}$ is not defended by $S$.

Proof of Theorem 5 Let us first consider the argument $i_{1}$. If $i_{1}=1$, then $M(A F)=$ $M\left(i_{1}, 2, \ldots, n\right)$. Otherwise, by making the dual interchange $1 \rightleftharpoons i_{1}$ to the matrix $M(A F)$ we get the matrix $M\left(i_{1}, \ldots, 1, \ldots\right)$, where 1 is at the position where $i_{1}$ is in the natural permutation.

Suppose the matrix $M\left(i_{1}, i_{2}, \ldots, i_{r-1}, p_{1}, p_{2}, \ldots, p_{n-r+1}\right)$ has been obtained, where

$\left(p_{1}, p_{2}, \ldots, p_{n-r+1}\right)$ is a permutation of the set $A \backslash\left\{i_{1}, i_{2}, \ldots, i_{r-1}\right\}$. If $i_{r}=p_{1}$, then the matrix $M\left(i_{1}, i_{2}, \ldots, i_{r-1}, p_{1}, \ldots, p_{n-k+1}\right)=M\left(i_{1}, i_{2}, \ldots, i_{r-1}, i_{r}, p_{2}, \ldots, p_{n-r+1}\right)$. Otherwise, there is some $1<l \leq n-r+1$ such that $i_{r}=p_{l}$. So, we can make the dual interchange $p_{l} \rightleftharpoons i_{r}$ to the matrix $M\left(i_{1}, i_{2}, \ldots, i_{r-1}, p_{1}, p_{2}, \ldots, p_{n-r+1}\right)$, and turn it into the matrix $M\left(i_{1}, i_{2}, \ldots, i_{r}, p_{1}, \ldots, p_{l-1}, \ldots, p_{l+1}, \ldots, p_{n-r+1}\right)$.

By mathematical induction, the first part has been proved.

Let $M(1,2, \ldots, n)=\left(a_{s, t}\right)$ and $M\left(i_{1}, i_{2}, \ldots, i_{k}, j_{1}, j_{2}, \ldots, j_{h}\right)=\left(b_{s, t}\right)$, then it is easy to check that $a_{i_{s}, i_{t}}=b_{s, t}(1 \leq s, t \leq k), a_{j_{p}, j_{q}}=b_{k+p, k+q}(1 \leq p, q \leq h)$, and $a_{i_{s}, j_{p}}=b_{s, k+p}(1 \leq$ $s \leq k, 1 \leq p \leq h)$. Therefore,

$$
M\left(i_{1}, i_{2}, \ldots, i_{k}, j_{1}, \ldots, j_{h}\right)=\left(\begin{array}{cc}
M^{c f}(S) & M^{s}(S) \\
M^{a}(S) & M^{c}(S)
\end{array}\right)
$$

where $M^{c f}(S), M^{s}(S), M^{a}(S)$ and $M^{c}(S)$ are the $c f$-subblock, $s$-subblock, $a$-subblock and $c$-subblock of $S$ in the matrix $M(A F)$, respectively.

Proof of Theorem 6: By Corollary 1, we can make a sequence of dual interchanges to $M(A F)$ and turn it into the matrix

$$
M\left(i_{1}, i_{2}, \ldots, i_{k}, j_{1}, \ldots, j_{h}\right)=\left(\begin{array}{cc}
O & M^{s}(S) \\
M^{a}(S) & M^{c}(S)
\end{array}\right)
$$

where $M^{s}(S), M^{a}(S)$ and $M^{c}(S)$ are the $s$-subblock, $a$-subblock and $c$-subblock of $S$ in the matrix $M(A F)$, respectively.

If there is no zero column in the subblock $M^{s}(S)$ of $M\left(i_{1}, i_{2}, \ldots, i_{k}, j_{1}, \ldots, j_{h}\right)$, then $q=0$ and we have done. Otherwise, we may assume that $M_{*, t_{1}}^{s}, \ldots, M_{*, t_{q}}^{s}(1 \leq q \leq h)$ are all the zero column vectors in $M^{s}(S)$. Certainly, they correspond to the columns $k+t_{1}, k+t_{2}$, $\ldots, k+t_{q}$ of the matrix $M\left(i_{1}, i_{2}, \ldots, i_{k}, j_{1}, \ldots, j_{h}\right)$ respectively.

Similar as in Theorem 5 , by making a sequence of dual interchanges to $M\left(i_{1}, i_{2}, \ldots\right.$, $\left.i_{k}, j_{1}, \ldots, j_{h}\right)$ we can turn it into the matrix $M\left(i_{1}, i_{2}, \ldots, i_{k}, j_{t_{1}}, \ldots, j_{t_{q}}, j_{s_{1}}, \ldots, j_{s_{l}}\right)$, where $\left(j_{s_{1}}, \ldots, j_{s_{l}}\right)$ is a permutation of $A \backslash\left\{i_{1}, i_{2}, \ldots, i_{k}, j_{t_{1}}, \ldots, j_{t_{q}}\right\}$. It is easy to see that the matrix $M\left(i_{1}, i_{2}, \ldots, i_{k}, j_{t_{1}}, \ldots, j_{t_{q}}, j_{s_{1}}, \ldots, j_{s_{l}}\right)$ has the following form 


$$
\left(\begin{array}{ccc}
O_{k, k} & O_{k, q} & S_{k, l} \\
A_{q, k} & C_{q, q} & E_{q, l} \\
F_{l, k} & G_{l, q} & H_{l, l}
\end{array}\right),
$$

where each column vector of $S_{k, l}$ is nonzero, $k+q+l=n$ and $q \geq 1$.

Proof of Theorem 7 Without lost of generality, we can assume that $i_{s}=s$ for each $1 \leq s \leq k$. Let $M(A F)$ be the matrix of $A F$, and the matrix $M_{r 1}(A F)$ be the reduced matrix with respect to $S$.

If $S$ is stable in $A F$, then every column vector of $M^{s}(S)$ is nonzero. It follows that every column vector of $M_{r 1}(\{t\})$ is nonzero, since $M_{r 1}(\{t\})$ is the sum of all rows of $M^{s}(S)$. By Theorem 2, $\{t\}$ is stable in the reduced argumentation framework.

Conversely, if $\{t\}$ is table in $\left(B 1, R_{r 1}\right)$, then every column vector of $M_{r 1}(\{t\})$ is nonzero. Therefore, every column vector of $M^{s}(S)$ is nonzero because of the same reason as above. So, $S$ is stable in $A F$ by Theorem 2.

Proof of Theorem 10 We apply the Corollary 3 to $A F$ and turn $M(A F)$ into the matrix $M\left(i_{1}, \ldots, i_{k}, j_{t_{1}}, \ldots, j_{t_{l}}, j_{s_{1}}, \ldots, j_{s_{m}}\right)$

$$
=\left(\begin{array}{ccc}
O_{k, k} & O_{k, l} & S_{k, m} \\
O_{l, k} & C_{l, l} & E_{l, m} \\
F_{m, k} & G_{m, l} & H_{m, m}
\end{array}\right),
$$

where the column vectors of $S_{k, m}$ are all nonzero, $S=\left\{i_{1}, \ldots, i_{k}\right\}$ and $k+l+m=n$. Let $B=\left\{j_{t_{1}}, \ldots, j_{t_{l}}\right\}$, we apply the corollary again to $\left.A F\right|_{B}$ and turn $C_{l, l}$ into the matrix $M\left(p_{1}, \ldots, p_{u}, q_{r_{1}}, \ldots, q_{r_{v}}, q_{h_{1}}, \ldots, q_{h_{w}}\right)$.

$$
=\left(\begin{array}{ccc}
O_{u, u} & O_{u, v} & S_{u, w} \\
O_{v, u} & C_{v, v} & E_{v, w} \\
F_{w, u} & G_{w, v} & H_{w, w}
\end{array}\right),
$$

where the column vectors of $S_{u, w}$ are all nonzero, $T=\left\{p_{1}, \ldots, p_{u}\right\}$ and $u+v+w=l$.

According to the dual interchanges made to $C_{l, l}$, we make the corresponding dual interchanges to $M\left(i_{1}, \ldots, i_{k}, j_{t_{1}}, \ldots, j_{t_{l}}, j_{s_{1}}, \ldots, j_{s_{m}}\right)$ and turn it into the matrix

$$
\begin{aligned}
& M\left(i_{1}, \ldots, i_{k}, p_{1}, \ldots, p_{u}, q_{r_{1}}, \ldots, q_{r_{v}}, q_{h_{1}}, \ldots, q_{h_{w}}, j_{s_{1}}, \ldots, j_{s_{m}}\right) \\
&=\left(\begin{array}{cccccc}
O_{k, k} & O_{k, u} & O_{k, v} & O_{k, w} & S_{k, m} \\
O_{u, k} & O_{u, u} & O_{u, v} & S_{u, w} & E_{u, m} \\
O_{v, k} & O_{v, u} & C_{v, v} & E_{v, w} & E_{v, m} \\
O_{w, k} & F_{w, u} & G_{w, v} & H_{w, w} & E_{w, m} \\
F_{m, k} & G_{m, u} & G_{m, v} & G_{m, w} & H_{m, m}
\end{array}\right),
\end{aligned}
$$


where the column vectors of $S_{u, w}$ and $S_{k, m}$ are all nonzero, and $k+u+v+w+m=n$. By the same corollary, we conclude that $S \cup T$ is an admissible extension of $A F$.

Proof of Theorem 11 Sufficiency: Since $E_{1}$ is an admissible extension of $\left.A F\right|_{U}$, it is also admissible in $A F$ by the directionality principle. Therefore, $\left.A F\right|_{T}$ is the remaining sub-argumentation wrt $E_{1}$. Note that $E_{2}$ is an admissible extension in $\left.A F\right|_{T}$, we conclude that $E=E_{1} \cup E_{2}$ is admissible in $A F$ according to Theorem 10 .

Necessity: Let $E$ be an admissible extension of $A F$, then $E \cap U$ is admissible in $\left.A F\right|_{U}$ according to the directionality. Let $E_{1}=E \cap U=\left\{i_{1}, \ldots, i_{k}\right\}, R^{+}\left(E_{1}\right)=\left\{p_{1}, \ldots, p_{s}\right\}$, $E_{2}=E \backslash E_{1}=\left\{j_{1}, \ldots, j_{h}\right\}, R^{+}\left(E_{2}\right)=\left\{q_{1}, \ldots, q_{t}\right\}$ and $A \backslash\left(E \cup R^{+}(E)\right)=\left\{l_{1}, \ldots, l_{r}\right\}$, then by a sequence of dual interchanges we can turn $M(A F)$ into the following matrix

$$
M\left(i_{1}, \ldots, i_{k}, j_{1}, \ldots, j_{h}, l_{1}, \ldots, l_{r}, p_{1}, \ldots, p_{h}, q_{1}, \ldots, q_{t}\right)=\left(\begin{array}{ccccc}
O_{k, k} & O_{k, h} & O_{k, r} & S_{k, p} & S_{k, q} \\
O_{h, k} & O_{h, h} & O_{h, r} & O_{h, p} & S_{h, q} \\
O_{r, k} & O_{r, h} & C_{r, r} & E_{r, p} & E_{r, q} \\
F_{p, k} & F_{p, h} & G_{p, r} & H_{p, p} & E_{p, q} \\
O_{q, k} & G_{q, h} & G_{q, r} & O_{q, p} & H_{q, q}
\end{array}\right) .
$$

Therefore, the matrix of $\left.A F\right|_{A \backslash\left(E_{1} \cup R^{+}\left(E_{1}\right)\right)}$ is

$$
M\left(j_{1}, \ldots, j_{h}, l_{1}, \ldots, l_{r}, q_{1}, \ldots, q_{t}\right)=\left(\begin{array}{ccc}
O_{h, h} & O_{h, r} & S_{h, q} \\
O_{r, h} & C_{r, r} & E_{r, q} \\
G_{q, h} & G_{q, r} & H_{q, q}
\end{array}\right) .
$$

According to Corollary $3, E_{2}=E \backslash E_{1}=\left\{j_{1}, \ldots, j_{h}\right\}$ is admissible in $\left.A F\right|_{A \backslash\left(E_{1} \cup R\left(E_{1}\right)\right)}$.

\section{B References}

1. P. Baroni, M. Giacomin, G. Guida, SCC-recursiveness: a general schema for argumentation semantics, Artificial Intelligence 168 (1-2) (2005) 162-210.

2. P. Baroni, M. Giacomin, On principle-based evaluation of extension-based argumentation semantics, Artificial Intelligence 171 (2007) 675-700.

3. P. Baroni, M. Giacomin, Skepticism relations for comparing argumentation semantics, International Journal of Approximate Reasoning 50 (2009) 854-866.

4. T. J. M. Bench-Capon, Paul E. Dunne, Argumentation in artificial intelligence, Artificial intelligence 171(2007)619-641

5. G. Boella, S. Kaci, L. van der Torre, Dynamics in argumentation with single extensions: abstraction principles and the grounded extension, in: Proceedings of the ECSQARU 2009, in: LNAI, vol. 5590, Springer-Verlag, Berlin/Heidelberg, 2009, pp. $107-118$. 
6. G. Boella, S. Kaci, L. van der Torre, Dynamics in argumentation with single extensions: attack refinement and the grounded extension, in: Proceedings of the AAMAS 2009, Budapest, Hungary, 2009, pp. 1213-1214.

7. M. Caminada, Semi-stable semantics, in: P.E. Dunne, T. Bench-Capon (Eds.), Proceedings of the First International Conference on Computational Models of Argument (COMMA 2006), IOS Press, Liverpool, UK, 2006, pp. 121-130.

8. M. Caminada, An algorithm for computing semi-stable semantics, in: K. Mellouli (Ed.), Proceedings of the ECSQARU 2007, in: LNAI, vol. 4724, Springer-Verlag, Berlin/Heidelberg, 2007, pp. 222-234.

9. M. Capobianco, C.I. Chesnevar, G.R. Simari, Argumentation and the dynamics of warranted beliefs in changing environments, JAAMAS 11 (2) (2005) 127-151.

10. D.V. Carbogim, Dynamics on formal argumentation, PhD thesis, University of Edinburgh, 2000.

11. C. Cayrol, F.D. de St-Cyr, M. Lagasquie-Schiex, Revision of an argumentation system, in: Proceedings of the KR2008, Sydney, Australia, 2008, pp. 124-134.

12. C. Cayrol, F.D. de Saint-Cyr, M. Lagasquie-Schiex, Change in abstract argumentation frameworks: adding an argument, Journal of Artificial Intelligence Research 38 (2010) 49-84.

13. Y.Dimopoulos, A. Torres, Graph theoretical structures in logic programs and default theories, Teoret. Comput. Sci. 170(1996)209-244.

14. P.M. Dung, On the acceptability of arguments and its fundamental role in nonmonotonic reasoning, logic programming and $n$-person games, Artificial Intelligence 77 (1995), 321-357.

15. P.E.Dunne, Computational properties of argument systems satisfying graph-theoretic constrains, Artificial Intelligence 171 (2007), 701-729.

16. P.E. Dunne, T.J.M. Bench-Capon, Complexity and combinatorial properties of argument systems, Technical report, University of Liverpool, 2001.

17. M.A. Falappa, A.J. Garca, G.R. Simari, Belief dynamics and defeasible argumentation in rational agents, in: Proceedings of the NMR2004, Whistler, BC, Canada, 2004, pp. 164-170.

18. Dov M. Gabbay, Introducing Equational Semantics for Argumentation Networks, in: Lecture Notes in Computer Science 6717, 2011, 19-35 
19. B. Liao, J. Li, R. Koons, Dynamics of argumentation systems: A division-based method, Artificial Intelligence 175 (2011) 1790-1814.

20. S. Modgil, Reasoning about preferences in argumentation frameworks, Artificial Intelligence 173 (2009) 901-934.

21. S. Modgil, M. Caminada, Proof theories and algorithms for abstract argumentation frameworks, in: I. Rahwan, G.R. Simari (Eds.), Argumentation in Artificial Intelligence, Springer, 2009, pp. 105-129.

22. E. Oikarinen, S.Woltran, Characterizing strong equivalence for argumentation frameworks, Artificial intelligence(2011), doi:10.1016/j.artint.2011.06.003.

23. I. Rahwan, Guillermo R. Simari, Argumentation in artificial intelligence, 2009.

24. N.D. Rotstein, M.O. Moguillansky, A.J. Garca, G.R. Simari, An abstract argumentation framework for handling dynamics, in: Proceedings of the NMR2008, Sydney, Australia, 2008, pp. 131-139.

25. G. Vreeswijk, Abstract argumentation system, Artificial intelligence 90(1997)225-279.

26. B. Verheij, A labeling approach to the computation of credulous acceptance in argumentation, in: Proceedings of the IJCAI-07, Hyderabad, India, 2007, pp. 623-628. 